\title{
ADOP in closed form for a hierarchy of multi-frequency single-baseline GNSS models
}

\author{
D. Odijk · P. J. G. Teunissen
}

Received: 7 August 2007 / Accepted: 7 November 2007 / Published online: 29 January 2008

(C) The Author(s) 2008

\begin{abstract}
Successful carrier phase ambiguity resolution is the key to high-precision positioning with Global Navigation Satellite Systems (GNSS). The ambiguity dilution of precision (ADOP) is a well-known scalar measure which can be used to infer the strength of the GNSS model for carrier phase ambiguity resolution. In this contribution we present analytical closed-form expressions for the ADOP. This will be done for a whole class of different multifrequency single baseline models. These models include the geometry-fixed, the geometry-free and the geometry-based models, respectively. And within the class of geometry-based models, we discriminate between short and long observation time spans, and between stationary and moving receivers. The easy-to-use ADOP expressions can be applied to infer the contribution of various GNSS model factors. They comprise, for instance, the type, the number and the precision of the GNSS observations, the number and selection of frequencies, the presence of atmospheric disturbances, the length of the observation time span and the length of the baseline.
\end{abstract}

Keywords ADOP · GNSS · GPS · Ambiguity resolution

\section{Introduction}

Crucial to precise (mm-cm level) GNSS positioning is the resolution of the integer carrier-phase ambiguities. For

D. Odijk (凶) • P. J. G. Teunissen

Delft Institute of Earth Observation and Space Systems (DEOS), Delft University of Technology, P.O. Box 5058,

2600 GB Delft, The Netherlands

e-mail: d.odijk@tudelft.nl

P. J. G. Teunissen

e-mail: p.j.g.teunissen@tudelft.nl this purpose, the integer least-squares principle embodied in the LAMBDA method has been proven to be optimal in the sense of maximizing the probability of correct integer estimation (Teunissen 1999), if the random errors of the measurements are assumed to have an elliptically contoured distribution. The normal distribution is the most well known member of the family of elliptically contoured distributions (Chmielewsky 1981). A high ambiguity success rate is obtained when the precision of the float ambiguity estimates is sufficiently high. In order to get insight into the quality of the ambiguities the ambiguity dilution of precision (ADOP) can be used. The ADOP, first introduced in Teunissen (1997b), is an intrinsic measure for the average precision of the ambiguities.

Since its introduction, the ADOP concept has been used in a wide scala of GPS applications. For example, Wu (2003) and Skaloud (1998) considered ADOPs in case (single-frequency) GPS data are integrated with INS data. Scherzinger $(2000,2001)$ used the ADOP concept to examine the impact of inertial aiding on RTK ambiguity precision during GPS outages. Lee et al. $(2002,2005)$ investigated the effect on ADOP of integrating GPS with pseudolites and inertial navigation systems. Wang et al. (2004) used the ADOP concept to infer the performance of an adaptive stochastic modeling technique for network-based RTK positioning. Chen et al. (2004) computed ADOPs to compare different signal scenarios for network RTK in the presence of Galileo and modernized GPS systems, while Vollath et al. (2003) analyzed — by means of ADOP — the impact of a fourth Galileo frequency on ambiguity resolution. In Ji et al. (2007) the ADOP is used to compute the ambiguity success rate which is used as criterion for the selection of independent combinations of Galileo frequencies for the CAR (cascading ambiguity resolution) method. Finally, Moore et al. (2003) considered ADOP in case of attitude 
determination of unmanned airborne vehicles, while Barrena and Colmenarejo (2002) computed ADOPs for generic formation flying scenarios.

It is possible to derive, easy-to-use, analytical closed-form expressions for the ADOP. These expressions enable one to infer the contribution of the measurement set-up or mathematical model to the precision of the ambiguities, without having to compute the ambiguity variance-covariance (vc-) matrix explicitly. They give a deeper insight in the various factors contributing to ADOP, in a qualitative as well as a quantitative sense. These contributing factors are, among others, the number of satellites, receiver-satellite geometry, observable types, precision of the observables, number of frequencies, length of observation time span, number of samples used and the in(ex)clusion of ionospheric delays. In Teunissen and Odijk (1997) already such closed-form ADOP expressions have been derived, however these are restricted to the geometry-free GPS model, the model which dispenses the relative receiver-satellite geometry. In Teunissen (1997b) expressions for the geometry-based model, the usual model for positioning, were derived, but only applicable for sufficient short baselines for which the relative ionospheric delays may be neglected. In this paper we will provide closedform expressions for a wide variety of single-baseline GNSS models.

This contribution is organized as follows. In Sect. 2, we describe the general structure of the multi-frequency, singlebaseline ionosphere-weighted GNSS model. This model applies to any GNSS for which ambiguity resolution is possible, such as GPS, modernized GPS and Galileo. It forms the basis for our closed-form derivations of the ADOP. The model is formulated such that, through its structure, a variety of different single-baseline models can be covered in a systematic way. In Sect. 2, we also give a description of the stochastic model and its structure. Apart from the standard form, it also allows the inclusion of cross-correlation, temporal correlation and satellite-dependent weighting (e.g. as function of satellite elevation). Section 3 starts with a brief description on the different parameter estimation steps for precise GNSS positioning. After that the definition and properties of the ADOP are given. The analytical closed-form expressions of the ADOP are given in Sects. 4, 5 and 6. They are given for a hierarchy of multi-frequency single baseline models. These models include the geometry-fixed, the geometry-free and the geometry-based models, and within the class of geometry-based models, the short and long observation time spans, and the cases of having stationary or moving receivers, are also covered. In order not to distract from the main results, detailed derivations of the closedform expressions can be found through a website, see MGP (2007).
Table 1 Overview of GNSS frequencies and wavelengths $\left(\lambda_{j}\right)$

\begin{tabular}{|c|c|c|c|c|c|c|}
\hline \multirow{3}{*}{$\frac{j}{1}$} & \multicolumn{3}{|c|}{ GPS } & \multicolumn{3}{|c|}{ Galileo (envisioned) } \\
\hline & \multicolumn{2}{|c|}{ Frequency (MHz) } & \multirow{2}{*}{$\frac{\lambda_{j}(\mathrm{~m})}{0.1903}$} & \multicolumn{2}{|c|}{ Frequency $(\mathrm{MHz})$} & \multirow{2}{*}{$\frac{\lambda_{j}(\mathrm{~m})}{0.1903}$} \\
\hline & L1 & 1575.42 & & L1 & 1575.42 & \\
\hline 2 & L2 & 1227.60 & 0.2442 & E5a & 1176.45 & 0.2548 \\
\hline 3 & L5 & 1176.45 & 0.2548 & E6 & 1278.75 & 0.2344 \\
\hline
\end{tabular}

\section{The ionosphere-weighted GNSS model}

\subsection{Observation equations}

Starting point is the GNSS model of carrier phase and code observation equations for a relative measurement set up: from two receivers (a single baseline) observations from at least two satellites are tracked. In a double-differenced (DD) form, they read as follows for a receiver-satellite combination $r-s$ relative to pivot receiver 1 and pivot satellite 1 at observation epoch $i$ and on frequency $j$, in units of meters, see e.g., Teunissen and Kleusberg (1998):

$$
\begin{aligned}
& \phi_{1 r, j}^{1 s}(i)=\ell_{1 r}^{1 s}(i)+t_{1 r}^{1 s}(i)-\frac{\lambda_{j}^{2}}{\lambda_{1}^{2}} l_{1 r, 1}^{1 s}(i)+\lambda_{j} M_{1 r, j}^{1 s}+\epsilon_{\phi_{1 r, j}^{1 s}(i)} \\
& p_{1 r, j}^{1 s}(i)=\ell_{1 r}^{1 s}(i)+t_{1 r}^{1 s}(i)+\frac{\lambda_{j}^{2}}{\lambda_{1}^{2}} l_{1 r, 1}^{1 s}(i)+\epsilon_{p_{1 r, j}^{1 s}(i)}
\end{aligned}
$$

where $\phi_{1 r, j}^{1 s}(i)$ and $p_{1 r, j}^{1 s}(i)$ denote the DD phase and code observable respectively, $\ell_{1 r}^{1 s}(i)$ the DD receiver-satellite range, $t_{1 r}^{1 s}(i)$ the DD tropospheric delay, $l_{1 r, 1}^{1 s}(i)$ the (firstorder) DD ionospheric delay on the first frequency, $\lambda_{j}$ the wavelength corresponding to the $j$ th frequency, $M_{1 r, j}^{1 s}$ the integer DD phase ambiguity, and $\epsilon_{\phi_{1 r, j}^{1 s}(i)}$ and $\epsilon_{p_{1 r, j}^{1 s}(i)}$ the random errors (measurement noise) for the DD phase and code observations, respectively. Since the ionospheric delays are frequency-dispersive, we may write the delays of all involved frequencies as function of the delay on the first frequency. In Table 1 an overview is given of the frequencies and wavelengths of the signals of (modernized) GPS and the envisioned Galileo system.

Aside from the phase and code observables, we introduce a third group of observables, the ionospheric observables. Their observation equation reads:

$1_{1 r, 1}^{1 s}(i)=l_{1 r, 1}^{1 s}(i)+\epsilon_{1_{1 r, 1}^{1 s}(i)}$

where $1_{1 r, 1}^{1 s}(i)$ denotes the DD ionospheric observable and $\epsilon_{1_{1 r, 1}(i)}$ the random error of this observable. By including these observables it is possible to incorporate a priori information on the ionospheric delays, allowing for a flexible 
GNSS model applicable for a wide range of baseline lengths (see Sect. 2.3). This model will be referred to as the ionosphere-weighted GNSS model. A similar type of stochastic modeling of the ionospheric delays was already used by Bock et al. (1986). Other applications of the ionosphereweighted model can be found in, among others, Schaffrin and Bock (1988), Goad and Yang (1994), Schaer (1994), Teunissen (1998), Odijk (2000), Liu (2001), Milbert (2005), Odijk (2002), O'Keefe et al. (2005), Wu et al. (2004) and Richtert and El-Sheimy (2005).

\subsection{A hierarchy of single-baseline GNSS models}

In this contribution we consider a hierarchy of single-baseline models for which the ADOP will be derived. These models differ in their information content and as a consequence, they also differ in their strength for ambiguity resolution. The weakest model that we consider is the so-called geometryfree model. The observation equations of this model are the ones given in Eq. (1). Thus instead of parameterizing the DD ranges further into the baseline components, the observation equations of the geometry-free model remain parameterized in the unknown DD ranges. As a consequence the relative receiver-satellite geometry does not play a role and no information on the GNSS ephemeris is needed. In case of the geometry-free model, both receivers of the single baseline may be either stationary or in in motion. But note, since the ranges and tropospheric delays are not separably estimable in case of the geometry-free model, that these two parameters will have to be lumped together in one parameter, the troposphere-biased range.

We speak of a geometry-based model when the DD ranges are further parameterized in the three unknown baseline components. The observation equations of the geometry-based model are nonlinear, whereas those of the geometry-free model are linear. Hence, in order to obtain a linear model, the observation equations of the geometry-based model need to be linearized with respect to the baseline components. The relative receiver-satellite geometry, which enters when evaluating the partial derivatives of the linearization, plays an important role in the geometry-based model. Elements of this geometry also enter when the different tropospheric delays are mapped to a single tropospheric zenith delay. In our geometry-based models, we do not estimate the satellite positions. Hence, the satellite positions are held fixed at their a priori values. For this purpose the GNSS broadcast ephemeris may be used if the baselines are sufficiently short, otherwise it is necessary to use precise (IGS) orbits. In our evaluation of the geometry-based model, we make a distinction between short and long observation time spans. Furthermore, we make a distinction between the case of a moving receiver and the case of a stationary receiver. We define the observation time span to be short, if it is permitted

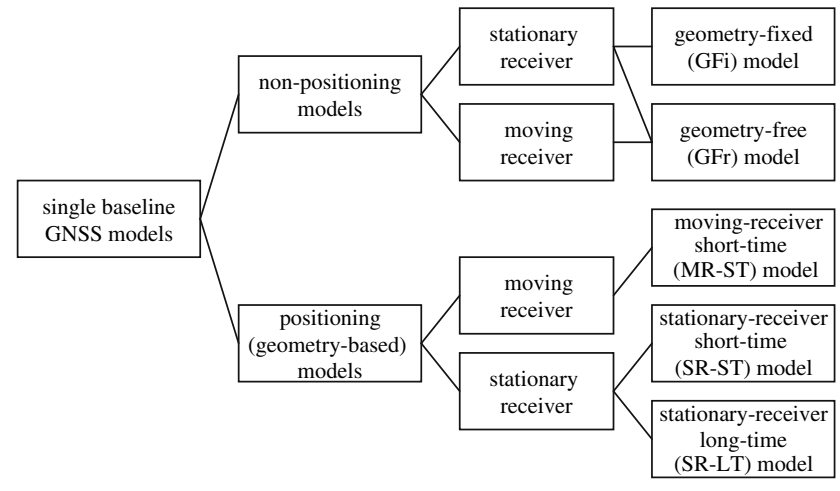

Fig. 1 A hierarchy of single-baseline GNSS models

to consider a 'frozen' relative receiver-satellite geometry, instead of a geometry which changes with the observation time span. It will be clear that the geometry-based model is a stronger model than the geometry-free model. The redundancy has increased, since now all DD ranges are coupled to the same three baseline components.

In addition to the geometry-free model and the geometrybased model, in this paper we also consider the geometryfixed model. This is the simplest and strongest of all models. It is the model in which the baseline parameters and the tropospheric zenith delay are assumed absent. Hence, as it is the case with the geometry-free model, the relative receiversatellite geometry does not play a role in the geometry-fixed model. But the reason is now that we assume the geometry known. Thus the only parameters that remain are the ambiguities and, possibly, the ionospheric delays.

Figure 1 depicts schematically the different versions of the single-baseline GNSS models considered in this paper. In the next section we describe in more detail the structure of the design matrices of these different GNSS models. Describing the similarities and differences in structure of the design matrices will help us in deriving and relating the various closed-form ADOP expressions in a systematic way.

\subsection{Observation model}

When it is assumed that GNSS data of $m$ satellites are simultaneously collected by two receivers on $j$ frequencies during a time span of $k$ epochs (with a constant sampling interval), then in a general form the following observation (or GaussMarkov) model can be formulated:

$E\{y\}=A_{1} x_{1}+A_{2} x_{2}+A_{3} x_{3}, \quad D\{y\}=Q_{y}$

In this model $E\{\cdot\}$ denotes the expectation operator and $D\{\cdot\}$ the dispersion operator, vector $y$ denotes the normally distributed GNSS data vector ('observed-minus-computed' in case of the linearized geometry-based model), $x_{i}, i=1,2,3$, the three parameter groups and $A_{i}, i=1,2,3$, their partial 
Table 2 Observables and variance matrix of the single-baseline model

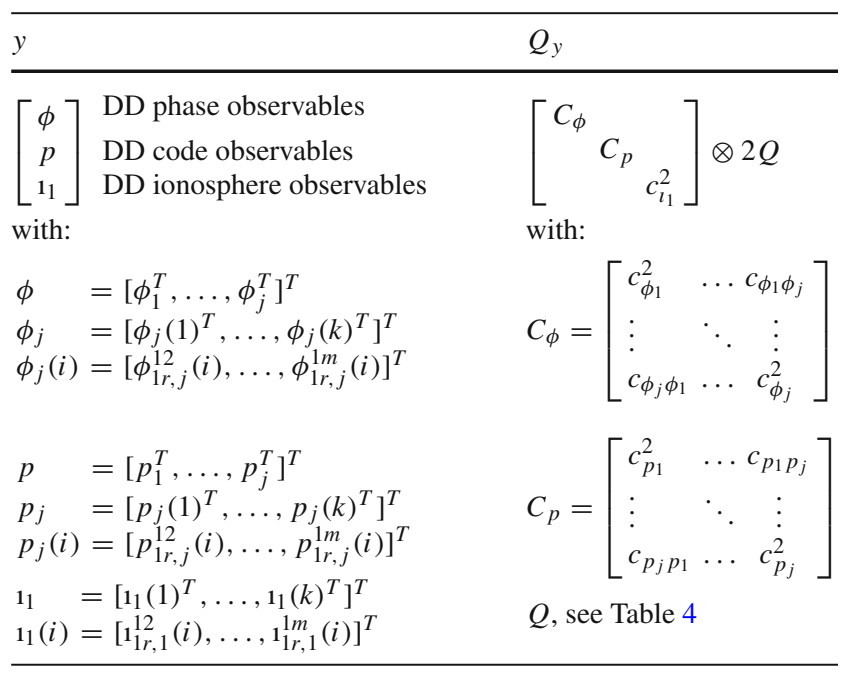

design matrices. The variance-covariance matrix $Q_{y}$ represents the stochastic properties of the observables.

\subsubsection{Observable types}

According to the observation equations given in Sect. 2.1, vector $y$ consists of three types of observables: the DD phase, code and ionospheric observables, generally denoted as $\phi, p$ and $1_{1}$. Table 2 explains how these observables are organized in vector $y$ and also the structure of vc-matrix $Q_{y}$ is given. In the notation of this matrix we use the Kronecker product $\otimes$ (Rao 1973), to keep the notation compact.

In the stochastic model, matrices $C_{\phi}$ and $C_{p}$ (both of dimension $j$ ) denote the cofactor matrices of the phase and code observables, respectively, whereas $c_{l_{1}}^{2}$ denotes the variance factor of the ionospheric observables. In these matrices $c_{\phi_{j}}^{2}$ and $c_{p_{j}}^{2}$ denote the variance factors of the phase and code observable, respectively, on the $j$ th frequency. By specifying the non-diagonal elements $c_{\phi_{1} \phi_{j}}$ and/or $c_{p_{1} p_{j}}$ one may (optionally) account for correlation between the observables on $j$ frequencies (also known as cross correlation). Concerning $c_{l_{1}}$, it holds for a parametrization of the ionospheric delays on the first frequency. If one would parameterize them on the $j$ th frequency instead, the following ionospheric variance factor is to be used: $c_{l_{j}}=\left(\lambda_{j}^{2} / \lambda_{1}^{2}\right) c_{l_{1}}$. The tuning of this ionospheric standard deviation is closely related to the length of the baseline: for short (a few km) baselines one usually sets it equal to zero $\left(c_{l_{1}}=0\right)$, since the relative ionospheric delays are usually such small that they may be neglected. As consequence the DD ionospheric parameters are removed. This (extreme) version of the ionosphereweighted model will be referred to as the ionosphere-fixed model. For longer baselines (e.g. up to a few tens of km's) one may have a priori ionospheric information available and depending on its quality one may set the ionospheric standard deviation $c_{l_{1}}$. For even longer baselines (e.g. up to a few hundred $\mathrm{km}$ ) usually no good a priori information is available and one wishes to use a model in which the ionospheric delays are purely estimated from the given phase and code data. Mathematically this corresponds to setting $c_{l_{1}}=\infty$ and this (extreme) version is referred to as the ionosphere-float model.

It is remarked that matrices $C_{\phi}$ and $C_{p}$ and factor $c_{l_{1}}^{2}$ are based on undifferenced observables, hence the factor 2 in Table 2 accounts for the differencing between the two receivers of the single baseline. The correlation due to the differencing between satellites is accounted for through matrix $Q$ in Table 2, which will be elaborated upon in Table 4.

\subsubsection{Parameter groups}

The three parameter groups of observation model (3) are the DD ambiguities, geometry parameters and the DD ionospheric delays. Table 3 explains their vector structure as well as their respective design matrices.

The first group, the DD ambiguities, contained in vector $a$, only apply to the phase data and are known to be integer and constant during the time span, provided that no cycle slips or loss-of-locks occur. Matrix $\Lambda$ contains the wavelengths, since the phase data are expressed in meters.

The second group, the geometry parameters, contained in vector $g$, depend on the single-baseline model and detailed
Table 3 Design matrices and parameters of the single-baseline model

\begin{tabular}{llll}
\hline & $\begin{array}{l}i=1 \\
\text { (ambiguities) }\end{array}$ & $\begin{array}{l}i=2 \\
\text { (geometry) }\end{array}$ & $\begin{array}{l}i=3 \\
\text { (ionospheric delays) }\end{array}$ \\
\hline \multirow{2}{*}{$A_{i}$} & {$\left[\begin{array}{c}\Lambda \\
0 \\
0\end{array}\right] \otimes\left(e_{k} \otimes I_{m-1}\right)$} & {$\left[\begin{array}{c}e_{j} \\
e_{j} \\
0\end{array}\right] \otimes B$} & {$\left[\begin{array}{c}-\mu \\
\mu \\
1\end{array}\right] \otimes\left(I_{k} \otimes I_{m-1}\right)$} \\
& with: & with: & with: \\
& $\Lambda=\operatorname{diag}\left(\lambda_{1}, \ldots, \lambda_{j}\right)$ & $B$, see Table 4 & $\mu=\left(\mu_{1}, \ldots, \mu_{j}\right)^{T}$ \\
& $e_{k}=(1, \ldots, 1)^{T}$ & and $\mu_{j}=\lambda_{j}^{2} / \lambda_{1}^{2}$
\end{tabular}


Table 4 Some matrices/vectors for the single-baseline model versions

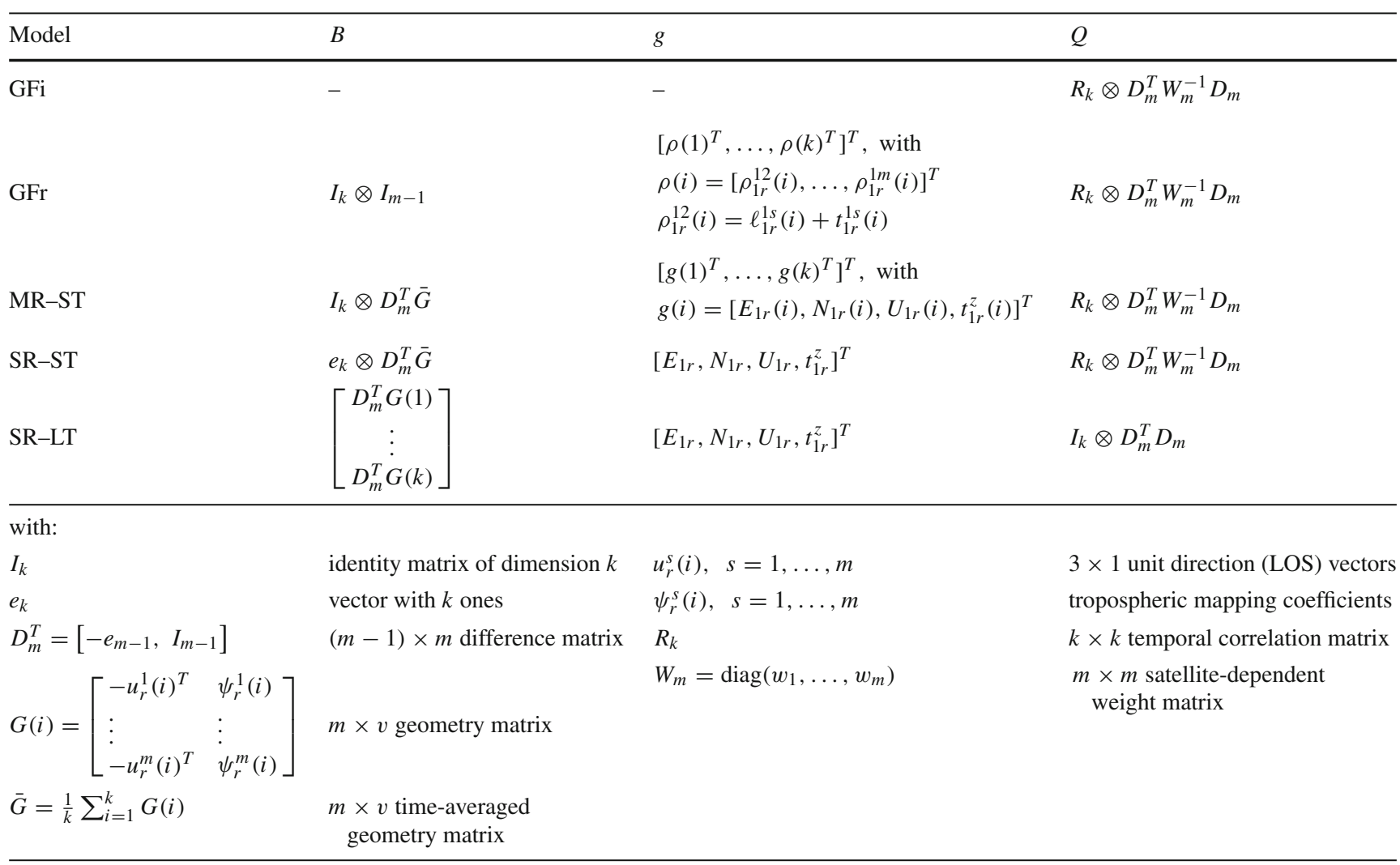

in Table 4. In case of the geometry-free model they consist of the troposphere-biased DD ranges, while for the three versions of the geometry-based model they consist of three coordinate components (e.g. in East-North-Up), plus (optionally) a relative tropospheric zenith delay parameter (if the observations are not fully a priori corrected for the troposphere using a troposphere model). The number of geometry parameters is generally denoted as $v$ and and can take on either $v=1$ (troposphere unknown, positions known), $v=3$ (troposphere known, positions unknown), or $v=4$ (troposphere unknown, positions unknown). In the partial design matrices for all three geometry-based models matrix $D_{m}^{T}$ denotes the between-satellite difference operator. In Table 4 it is defined using the first satellite as pivot or reference satellite, however, as it will be shown later, the results in terms of ADOP are invariant for the choice of pivot satellite. The matrix $G(i)$ appearing in the long-time geometry-based model captures the receiver-satellite unit direction (lineof-sight) vectors, plus, if necessary, tropospheric mapping coefficients of the $m$ satellites, see Table 4. For the two geometry-based models applying to short time spans instead of the changing geometry matrices $G(i)$ per epoch the average of all receiver-satellite geometry matrices over the observation time span is taken, which is denoted by matrix $\bar{G}$. Replacing the time-varying geometry with its time-averaged counterpart is permitted for a short time (e.g. a few minutes) since the GNSS constellation changes only slowly. The advantage of using such a 'frozen' geometry is that it facilitates the derivation of the closed-form ADOP expressions.

The last parameter group consists of the DD ionospheric delays on the first frequency, contained in vector $\iota_{1}$, see Table 3. Alternatively, we could have parameterized the ionospheric delays on the $j$ th frequency (denoted as $l_{j}$ ), however this does not affect ADOP. In that case instead of vector $\mu$ we would have to use another vector, say $\omega$, based on a parametrization of the ionospheric delays on the $j$ th frequency, where $\omega=\left(\lambda_{1}^{2} / \lambda_{j}^{2}\right) \mu$ and $\iota_{j}=\left(\lambda_{j}^{2} / \lambda_{1}^{2}\right) l_{1}$ (note that $\left.\omega \iota_{j}=\mu \iota_{1}\right)$.

\subsubsection{Stochastic model decomposition}

Table 4 also gives the structure of matrix $Q$, which is part of the stochastic model of the general single-baseline model. This matrix serves two goals: modeling of time correlation and/or satellite dependent weights. Temporal correlations between the observations of different epochs are taken into account by filling up the non-diagonal elements of the $k \times k$ matrix $R_{k}$ (this matrix has ones at its diagonal). However note from Table 4 that in case of the long-time geometrybased model these correlations are not accounted for $\left(R_{k}\right.$ is reduced to $I_{k}$ ). Reason for this is that with a long time span of data, temporal correlations are not an issue since they can be 
Table 5 Requirements to the ionosphere-weighted model with respect to number of observation epochs $(k)$, number of satellites $(m)$ and number of frequencies $(j)$. The number of baseline components $(v)$ is restricted to $v=1$ (only trop. zenith delay estimated), $v=3$ (only coordinates estimated), or $v=4$ (both coordinates and trop. zenith delay estimated)

\begin{tabular}{llll}
\hline Ionosphere-weighting & Model & Phase and code & Phase-only \\
\hline Ionosphere-fixed or-weighted & GFi & $k \geq 1, j \geq 1, m \geq 2$ & $k \geq 1, j \geq 1, m \geq 2$ \\
$0 \leq c_{l_{1}}<\infty$ & GFr & $k \geq 1, j \geq 1, m \geq 2$ & - \\
& MR-ST & $k \geq 1, j \geq 1, m \geq v+1$ & - \\
& SR-ST & $k \geq 1, j \geq 1, m \geq v+1$ & - \\
& SR-LT & $k \geq 1, j \geq 1, m \geq v+1$ & - \\
Gonosphere-float & GFi & $k \geq 1, j \geq 1, m \geq 2$ & - \\
$c_{l_{1}=\infty}=$ & $k \geq 1, j \geq 2, m \geq 2$ & - \\
& GFr & $k \geq 1, j \geq 2, m \geq v+1$ & - \\
& SR-ST & $k \geq 1, j \geq 2, m \geq v+1$ & - \\
& SR-LT & $\{k \geq 1, j \geq 2, m \geq v+1$ & -1 \\
& & & - \\
\hline
\end{tabular}

easily omitted by enlarging the sampling interval of the observations. For short-time applications however, an increase of the sampling interval may not be desirable or possible, so we may have to account for time correlation in the stochastic model. Through the $m \times m$-matrix $W_{m}$ (see Table 4 ) it is possible to weigh the observations depending on, for instance, the elevation of the satellite. However, this way of satellitedependent weighting is not accounted for in the closed-form expressions for the long-time geometry-based model, since it would make the derivations extremely difficult. For that model the satellite-dependent weights are assumed absent, i.e., $W_{m}=I_{m}$. In order to (partially) compensate for this deficiency, a suggestion idea would be to increase the phase and code standard deviations by a factor which is somewhat larger than 1 (for example 1.3), in case elevation dependency is suspected and the long-time geometry-based model is to be applied. It is finally emphasized that for both the geometry-fixed and geometry-free models matrix $Q$ as specified in Table 4 is to be used for short-time applications only.

\subsection{Requirements to the ionosphere-weighted model}

In order for the ionosphere-weighted model to be (uniquely) solvable, there are certain requirements to be met regarding the number of satellites, epochs, observable types and frequencies. These requirements are revealed by the specified structure of the different single-baseline models and are summarized in Table 5.

\section{The ADOP measure}

\subsection{Procedure to solve the GNSS model}

To solve the GNSS observation model as given in Eq. (3), we distinguish between integer parameters, i.e., the DD ambiguities contained in vector $a$, and real parameters, i.e., the range or baseline parameters and the DD ionospheric delays contained in vector $b$. Note that the dimension of the integer parameter vector is $j(m-1)$, but for sake of convenience this will be denoted as $n$, thus

$n=j(m-1) \Rightarrow a \in \mathbb{Z}^{n}$

In this paper the ionosphere-weighted GNSS model is solved in three steps, conform Teunissen (1993). An alternative two-step procedure is described in Xu et al. (1995). In the first step of the three-step procedure, we disregard the integer constraints $a \in \mathbb{Z}^{n}$ on the ambiguities and perform a standard least-squares adjustment. As a result, we obtain the (real-valued) estimates of $a$ and $b$, together with their vc-matrix:

$\left[\begin{array}{c}\hat{a} \\ \hat{b}\end{array}\right], \quad\left[\begin{array}{ll}Q_{\hat{a}} & Q_{\hat{a} \hat{b}} \\ Q_{\hat{b} \hat{a}} & Q_{\hat{b}}\end{array}\right]$.

This solution is referred to as the 'float' solution. In the second step, the float ambiguity estimate $\hat{a}$ is used to compute the corresponding integer least-squares ambiguity estimate, denoted as $\check{a}$ :

$\check{a}=S(\hat{a})$

with $S: \mathbb{R}^{n} \mapsto \mathbb{Z}^{n}$, the integer least-squares mapping from the $n$-dimensional space of reals to the $n$-dimensional space of integers. Once the integer ambiguities are computed, they are used in the third and final step to correct the float estimate of the real-valued parameters $b$. As a result we obtain the 'fixed' solution:

$\check{b}=\hat{b} \mid \check{a}=\hat{b}-Q_{\hat{b} \hat{a}} Q_{\hat{a}}^{-1}(\hat{a}-\check{a})$

If the ambiguity success rate, i.e., the probability that the estimated integers coincide with the true ambiguities, is sufficiently close to 1 , the precision of the fixed solution can be 
described by the following vc-matrix (in which the integer ambiguities are assumed non-stochastic):

$Q_{\hat{b} \mid a}=Q_{\hat{b}}-Q_{\hat{b} \hat{a}} Q_{\hat{a}}^{-1} Q_{\hat{a} \hat{b}}$

\subsection{Definition of ADOP}

The success of ambiguity resolution depends on the quality of the float ambiguity estimates: the more precise the float ambiguities, the higher the probability of estimating the correct integer ambiguities. For practical applications it would be helpful if, instead of having to evaluate all the entries of the ambiguity vc-matrix, one could work with an easyto-evaluate scalar precision measure. In Teunissen (1997b) the ADOP was introduced as such a measure. It is defined as:

$$
\mathrm{ADOP}=\left|Q_{\hat{a}}\right|^{1 /(2 n)}[\mathrm{cyc}]
$$

By taking the determinant of the float ambiguity vc-matrix a simple scalar is obtained, which not only depends on the variances of the ambiguities, but also on their covariances. By raising the determinant to the power $1 /(2 n)$, with $n$ the dimension of the vc-matrix, the scalar is, like the ambiguities, expressed in cycles.

It should be emphasized that the above definition of the ADOP measure differs from the traditional DOP or dilution of precision measures, such as the position (PDOP), the vertical (VDOP) or the horizontal dilution of precision (HDOP). These latter DOP measures are all based on the trace of the vc-matrix of the coordinates, instead of the determinant. We however believe that the trace cannot be used for the ambiguities, and this is motivated as follows. First, the trace of a vc-matrix is not invariant under ambiguity transformations, for example due to a change of reference satellite of the DD ambiguities. This is not an issue for the traditional DOP measures, since the choice of reference satellite does not affect the coordinate solution. For these coordinate-based DOP measures it is important that the trace remains invariant under orthogonal transformations, like a rotation of the coordinate frame of reference. A change of reference satellite is however not an orthogonal transformation. A second reason for not using the trace is that it does not take the correlation between ambiguities into account (since it is only based on the diagonal elements). However, it is known that the DD ambiguities can be highly correlated, especially in case of short observation times.

\subsection{Properties of ADOP}

The properties of the ADOP measure are briefly reviewed in this section.

First, the ADOP is invariant for the class of admissible ambiguity transformations. Suppose we transform the float ambiguities $\hat{a}$ to a vector $\hat{z}$ of the same dimension, i.e.,

$\hat{z}=Z^{T} \hat{a}, \quad Q_{\hat{z}}=Z^{T} Q_{\hat{a}} Z$,

then the transformation is admissible when the square matrix $Z$ fulfils two criteria (Teunissen 1993): (i) it should have integer entries, and (ii) it should be volume preserving, i.e., $|Z|= \pm 1$. Thus, $Z$ is a so-called unimodular matrix (Xu et al. 1995). It can be easily seen that the determinant of the transformed ambiguity vc-matrix remains invariant, since:

$\left|Q_{\hat{z}}\right|=\left|Z^{T} Q_{\hat{a}} Z\right|=|Z|^{T}\left|Q_{\hat{a}}\right||Z|=\left|Q_{\hat{a}}\right|$

Because of this property the ADOP remains the same, among others, under the transformation that corresponds to a change of reference satellite, and the decorrelating Z-transformation of the LAMBDA method. This transformation is carried out to enhance the search for the integer ambiguities.

A second property is that the ADOP is one-to-one related to the volume of the ambiguity search space. This $n$-dimensional search space is defined as $(\hat{a}-a)^{T} Q_{\hat{a}}^{-1}(\hat{a}-$ a) $\leq \chi^{2}$, with $\chi^{2}$ a scale factor, and its volume is computed as (Teunissen and Odijk 1997)

$V_{n}=\chi^{n} U_{n} \sqrt{\left|Q_{\hat{a}}\right|}=\chi^{n} U_{n} \mathrm{ADOP}^{n}$

where $U_{n}$ is the volume of the $n$-dimensional unit sphere. If the dimension is not changed, it can be easily seen that the search space shrinks when ADOP becomes smaller.

A third property of the ADOP is that it equals the geometric mean of the standard deviations of the ambiguities, in case the ambiguities are completely decorrelated. This follows from $\left|Q_{\hat{a}}\right|=\prod_{i=1}^{n} \sigma_{\hat{a}_{i}}^{2}\left|K_{\hat{a}}\right|$, where $\sigma_{\hat{a}_{i}}$ is the standard deviation of the $i$ th ambiguity and $K_{\hat{a}}$ the ambiguity correlation matrix. Since the LAMBDA method produces ambiguities that are largely decorrelated, the ADOP approximates the average precision of the transformed ambiguities.

Since the ADOP gives a good approximation to the average precision of the ambiguities, it also provides for a good approximation to the integer least-squares ambiguity success rate (Verhagen 2005, Ji et al. 2007). We therefore have the following approximation:

$$
P\left(\check{a}_{\mathrm{LS}}=a\right)=P\left(\check{z}_{\mathrm{LS}}=z\right) \simeq \underbrace{\left[2 \Phi\left(\frac{1}{2 \mathrm{ADOP}}\right)-1\right]^{n}}_{P_{\mathrm{ADOP}}}
$$

in which $\check{a}_{\text {LS }}$ and $\check{z}_{\text {LS }}$ are the integer least-squares ambiguity estimators of the original and transformed ambiguities, respectively, and where $\Phi(\cdot)$ is the standard normal cumulative distribution function, i.e., $\Phi(x)=\int_{-\infty}^{x} \frac{1}{\sqrt{2 \pi}} \exp \left\{-\frac{1}{2} v^{2}\right\} \mathrm{d} v$. Figure 2 shows $P_{\mathrm{ADOP}}$ as function of ADOP for varying levels of $n(n=1, \ldots, 20)$. It can be seen that the ADOPbased success rate decreases for increasing ADOP and this decrease is steeper the more ambiguities are involved. In general, Fig. 2 shows that if ADOP is smaller than about 


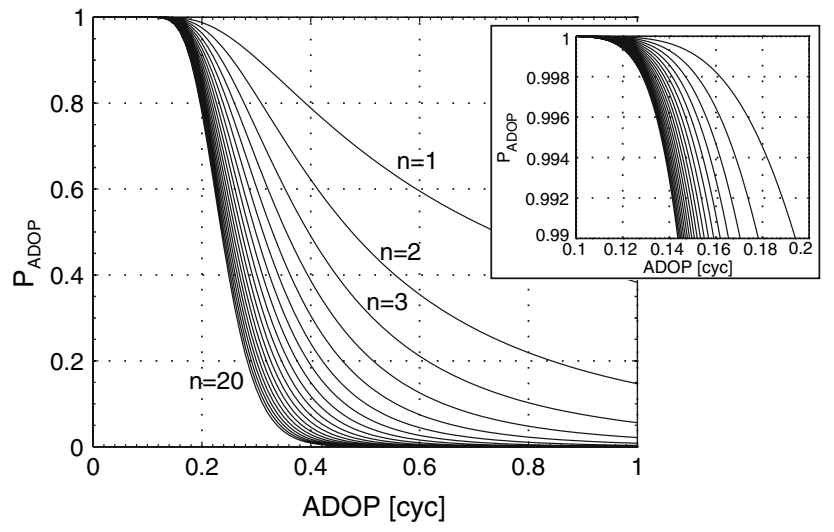

Fig. $2 P_{\mathrm{ADOP}}$ versus $\mathrm{ADOP}$ for varying number of DD ambiguities $n$

0.12 cyc, $P_{\text {ADOP }}$ becomes larger than 0.999 , while for ADOP smaller than 0.14 cyc, $P_{\text {ADOP }}$ is always better than 0.99 .

\subsection{Computing ADOP}

Although we will present easy-to-evaluate, closed-form expressions of the ADOP for a variety of GNSS models, there are still models for which one would need to evaluate the determinant of the ambiguity vc-matrix numerically in order to determine the ADOP value. For those cases one may either use the eigenvalues or the conditional variances obtained of the original or LAMBDA-transformed vc-matrix of the ambiguities. When the eigenvalues $\eta_{\hat{a}_{i}}$ of the ambiguity vc-matrix are used, we have:

$$
\mathrm{ADOP}=\prod_{i=1}^{n} \eta_{\hat{a}_{i}}^{\frac{1}{2 n}}
$$

Instead of working with eigenvalues, a cheaper way would be to make use of the conditional variances. This approach is based on using a triangular decomposition or a Cholesky decomposition of the ambiguity vc-matrix or its inverse. The entries of the diagonal matrix $D$ in the $L D L^{T}$-decomposition of the vc-matrix are the sequential conditional variances of the ambiguities. Since the determinant of the diagonal matrix $D$ equals the determinant of the vc-matrix, the ADOP becomes:

$$
\mathrm{ADOP}=\prod_{i=1}^{n} \sigma_{\hat{a}_{i \mid I}^{\frac{1}{n}}}^{\frac{1}{2}}
$$

The conditional variances $\sigma_{\hat{a}_{i \mid I}}^{2}$ are already available when the search for the integer least-squares ambiguities is based on a sequential conditional least-squares adjustment, as is the case with the LAMBDA method.

\subsection{Decomposition of ADOP expressions}

The starting point of the ADOP derivations is the expression for the vc-matrix of the real-valued parameters conditioned on the integer parameters, see Eq. (8). Using the determinant factorization rule, see e.g. Koch (1999), the determinant of this matrix can be written as:

$\left|Q_{\hat{b} \mid a}\right|=\frac{\left|Q_{\hat{b}}\right|}{\left|Q_{\hat{a}}\right|}\left|Q_{\hat{a}}-Q_{\hat{a} \hat{b}} Q_{\hat{b}}^{-1} Q_{\hat{b} \hat{a}}\right|$

At the right side of this expression - which was already found by Teunissen (1995) - the determinant of the ambiguity vcmatrix conditioned on the real parameters is recognized, i.e., $Q_{\hat{a} \mid b}=Q_{\hat{a}}-Q_{\hat{a} \hat{b}} Q_{\hat{b}}^{-1} Q_{\hat{b} \hat{a}}$. Using this, we may formulate the following expression for the determinant of the float ambiguity vc-matrix:

$\left|Q_{\hat{a}}\right|=\left|Q_{\hat{a} \mid b}\right| \frac{\left|Q_{\hat{b}}\right|}{\left|Q_{\hat{b} \mid a}\right|}$

Thus, the determinant of the ambiguity vc-matrix can be computed from the determinant of the ambiguity vc-matrix conditioned on the real parameters and the ratio of the determinants of the float and fixed real-valued parameters themselves. This latter ratio $\left|Q_{\hat{b}}\right| /\left|Q_{\hat{b} \mid a}\right|$ in fact measures the gain in precision due to ambiguity fixing. The reason for using the determinant expression in Eq. (17) to derive the closed-form ADOP expressions is that it is easier to derive expressions for the two separate parts $\left|Q_{\hat{a} \mid b}\right|$ and $\left|Q_{\hat{b}}\right| /\left|Q_{\hat{b} \mid a}\right|$, than for the total expression at once. To shorten the notation, all vc-matrices obtained with the ambiguities fixed will be denoted with a 'check' sign, e.g., $Q_{\breve{b}}=Q_{\hat{b} \mid a}$.

\section{Geometry-fixed ADOP expression}

In case of the geometry-fixed model the vector of real-valued parameters only consists of the DD ionospheric delays. Thus, $b=\imath_{1}$. Consequently, we need to evaluate the following determinantal expression, cf. Eq. (17):

$\left|Q_{\hat{a} \mid \rho}\right|=\left|Q_{\hat{a} \mid \rho, l_{1}}\right| \frac{\left|Q_{\hat{\imath}_{1} \mid \rho}\right|}{\left|Q_{\check{l}_{1} \mid \rho}\right|}$

where we used the subscript $\mid \rho$ to emphasize that in this model the geometry parameters (DD ranges) are fixed.

The determinant of the ambiguity vc-matrix conditioned on range and ionospheric parameters can be derived as the following analytical expression of the variables of the ionosphere-weighted model, see Sect. 2:

$\left|Q_{\hat{a} \mid \rho, l_{1}}\right|=\left[\frac{2^{j}\left|C_{\phi}\right|}{\prod_{i=1}^{j} \lambda_{i}^{2}}\right]^{m-1}\left[\left(\frac{1}{\left[e_{k}^{T} R_{k}^{-1} e_{k}\right]}\right)^{m-1} \frac{\sum_{s=1}^{m} w_{s}}{\prod_{s=1}^{m} w_{s}}\right]^{j}$

For a proof, we refer to MGP (2007). Note that $\left[e_{k}^{T} R_{k}^{-1} e_{k}\right]$ is a scalar, and this is emphasized by the square brackets. 
For the second part of the expression in Eq. (18) we need expressions for the float and fixed vc-matrices of the ionospheric parameters conditioned on the ranges. These are analytically derived as:

$$
\begin{aligned}
Q_{\hat{l}_{1} \mid \rho} & =2 c_{\check{\iota}_{1} \mid \rho}^{2}\left[R_{k}+\left(\frac{c_{\hat{i}_{1} \mid \rho}^{2}}{c_{i_{1} \mid \rho}^{2}}-1\right) \frac{1}{\left[e_{k}^{T} R_{k}^{-1} e_{k}\right]} e_{k} e_{k}^{T}\right] \otimes D_{m}^{T} W_{m}^{-1} D_{m} \\
Q_{\check{\iota}_{1} \mid \rho} & =2 c_{\check{\iota}_{1} \mid \rho}^{2} R_{k} \otimes D_{m}^{T} W_{m}^{-1} D_{m}
\end{aligned}
$$

Again, see MGP (2007) for a proof. In these expressions the variance factors of the float and fixed ionospheric delays conditioned on the ranges are computed as:

$$
c_{\hat{\imath}_{1} \mid \rho}^{2}=\frac{1}{\left[\mu^{T} C_{p}^{-1} \mu\right]+c_{l_{1}}^{-2}}, \quad c_{\check{\iota}_{1} \mid \rho}^{2}=\frac{1}{\left[\mu^{T}\left(C_{\phi}^{-1}+C_{p}^{-1}\right) \mu\right]+c_{l_{1}}^{-2}}
$$

When we take the ratio of the determinants of both float and fixed vc-matrices in Eq. (20), it can be easily seen that the variance factor $2 c_{\iota_{1} \rho}^{2}$ plus the determinant of $D_{m}^{T} W_{m}^{-1} D_{m}$ are eliminated in the ratio, since they appear exactly in the same way in both expressions. We thus arrive at:

$$
\frac{\left|Q_{\hat{\iota}_{1} \mid \rho}\right|}{\left|Q_{\iota_{1} \mid \rho}\right|}=\frac{\left|R_{k}+\left(\frac{c_{\hat{1}_{1} \mid \rho}^{2}}{c_{\grave{l}_{1} \mid \rho}^{2}}-1\right) \frac{1}{\left[e_{k}^{T} R_{k}^{-1} e_{k}\right]} e_{k} e_{k}^{T}\right|^{m-1}}{\left|R_{k}\right|^{m-1}}
$$

The numerator of this ratio can be simplified further, using the determinant factorization rule. We obtain:

$$
\frac{\left|Q_{\hat{\iota}_{1} \mid \rho}\right|}{\left|Q_{\check{\iota}_{1} \mid \rho}\right|}=\frac{\left|R_{k}\right|^{m-1}\left(c_{\hat{\imath}_{1} \mid \rho}^{2} / c_{\check{\iota}_{1} \mid \rho}^{2}\right)^{m-1}}{\left|R_{k}\right|^{m-1}}=\left[\frac{c_{\hat{\iota}_{1} \mid \rho}^{2}}{c_{\iota_{1} \mid \rho}^{2}}\right]^{m-1}
$$

Thus, the determinant ratio is only governed by the ratio of the float and fixed ionosphere variance factors conditioned on the ranges, and the number of satellites. Despite that time correlation, satellite-dependent weighting and number of samples have impact on the float and fixed vc-matrices themselves, see Eq. (20), they do not affect the ratio of their determinants. Since $c_{\hat{l}_{1} \mid \rho}^{2}$ is always larger than or equal to $c_{\tilde{l}_{1} \mid \rho}^{2}$, we may rewrite the ratio of float and fixed ionosphere variance factors conditioned on the ranges as a factor which is always larger than or equal to 1 :

$\frac{c_{\hat{1}_{1} \mid \rho}^{2}}{c_{\hat{i}_{1} \mid \rho}^{2}}=1+\frac{1}{\jmath}$

Scalar I will be referred to as the ionosphere factor. Now in the ionosphere-fixed $\left(c_{l_{1}}=0\right)$ case it holds that $I=\infty$ (and the ratio equals 1$)$, while in the ionosphere-float $\left(c_{l_{1}}=\infty\right)$ case it equals $]=\left[\mu^{T} C_{p}^{-1} \mu\right] /\left[\mu^{T} C_{\phi}^{-1} \mu\right]$.

Raising the determinant in Eq. (18) to the power $1 /(2 n)$, with $n=j(m-1)$, we arrive at the following ADOP expression for the geometry-fixed model:
Table 6 Symbols used in Figs. 3 to 8

\begin{tabular}{ll}
\hline Symbol & Meaning of symbol \\
\hline$j$ & Number of frequencies \\
$k$ & Number of observation epochs \\
$m$ & Number of satellites \\
$v$ & Number of baseline components \\
$c_{\phi}$ & Square root of variance factor of phase observables \\
$c_{p}$ & Square root of variance factor of code observables \\
$c_{l_{1}}$ & Square root of variance factor of ionosphere observables \\
$c$ & Correlation coefficient between dual-frequency data \\
$\alpha$ & Constant for satellite-dependent weighting (shows up in $w_{s}$ ) \\
$\beta$ & Temporal correlation coefficient (shows up in $R_{k}$ ) \\
\hline
\end{tabular}

$$
\begin{aligned}
& \mathrm{ADOP}^{\mathrm{GFi}} \\
& =\underbrace{\frac{\sqrt{2}\left|C_{\phi}\right|^{\frac{1}{2 j}}}{\check{\lambda}}}_{f_{1}} \underbrace{\left[\frac{1}{e_{k}^{T} R_{k}^{-1} e_{k}}\right]^{\frac{1}{2}}}_{f_{2}} \underbrace{\left[\frac{\sum_{s=1}^{m} w_{s}}{\prod_{s=1}^{m} w_{s}}\right]^{\frac{1}{2(m-1)}}}_{f_{3}} \underbrace{\left[1+\frac{1}{\bar{J}}\right]^{\frac{1}{2 j}}}_{f_{4}}
\end{aligned}
$$

with $\breve{\lambda}=\prod_{i=1}^{j} \lambda_{i}^{1 / j}$ the geometric mean of the wavelengths. Thus, the geometry-fixed ADOP can be written as a product of four (dimensionless) factors. In the following subsections we study the impact of each factor $f_{i}, i=1, \ldots, 4$ in more detail. The sensitivity of the geometry-fixed ADOP to changes in the model will be analyzed by means of the graphs depicted in Fig. 3. All graphs apply to GPS. In Table 6 we recapitulate the symbols used in the graphs in Fig. 3 (these symbols are also used in the forthcoming Figs. 4 to 8).

\subsection{Cross correlation (and phase/code standard deviations)}

In absence of cross correlation the full phase vc-matrix (see Table 2) reduces to $C_{\phi}=\operatorname{diag}\left(c_{\phi_{1}}^{2}, \ldots, c_{\phi_{j}}^{2}\right)$. Then $\left|C_{\phi}\right|^{\frac{1}{2 j}}=$ $\prod_{i=1}^{j} c_{\phi_{i}}^{1 / j}=\breve{c}_{\phi}$, i.e., the geometric mean of the phase standard deviations. Factor $f_{1}$ then reduces to $\sqrt{2} \breve{c}_{\phi} / \breve{\lambda}$, from which easily follows that ADOP benefits from high-quality phase data with long wavelengths. If we assume equal standard deviations for all phase observables, the geometric mean becomes simply $\breve{c}_{\phi}=c_{\phi}$. In case of GPS, if $c_{\phi}=3 \mathrm{~mm}$, factor $f_{1} \simeq 0.02$, irrespective of the number of frequencies used. Now suppose that we have phase observables on two frequencies that are cross correlated, having the following cofactor matrix

$C_{\phi}=c_{\phi}^{2}\left[\begin{array}{ll}1 & c \\ c & 1\end{array}\right], \quad-1<c<1$

where $c$ is the correlation coefficient between the two frequencies. We then have $\left|C_{\phi}\right|^{\frac{1}{2 j}}=c_{\phi}\left(1-c^{2}\right)^{1 / 4}$. In Fig. 3 
Fig. 3 Sensitivity of geometry-fixed ADOPs to changes in the model. For the three graphs at top it holds that $k=1, j=2$ and $m=6$, while for the three graphs at bottom it holds that $c_{\phi}=0.003 \mathrm{~m}, c_{p}=$ $0.30 \mathrm{~m}$ and $c_{l_{1}}=\infty$
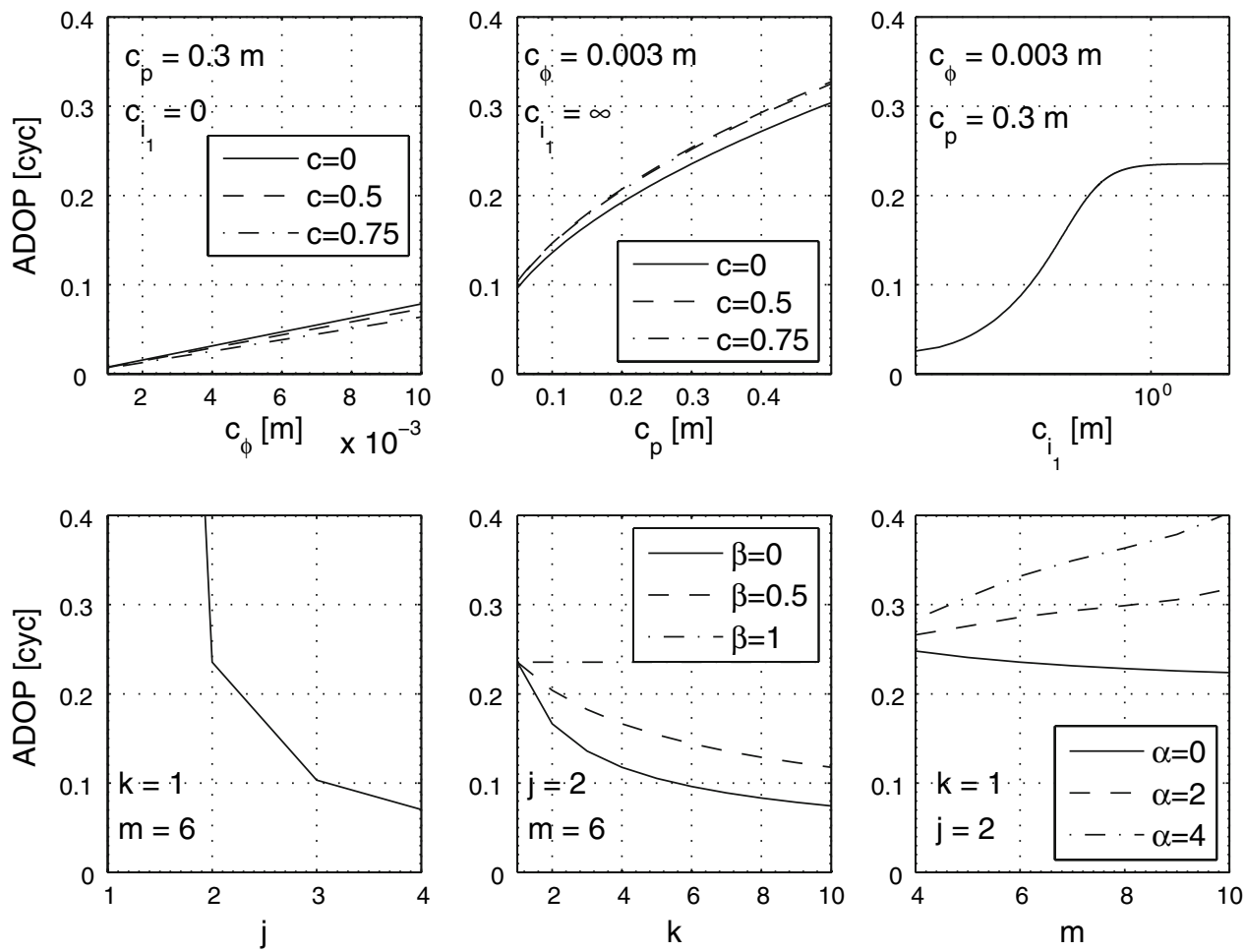

(top left), the geometry-fixed is plotted (for $k=1, m=6$ and $W_{m}=I_{m}$ ) as function of $c_{\phi}$ for three correlation coefficients: $c=0$ (no cross correlation), $c=0.5$ and $c=0.75$. This is done for the ionosphere-fixed $\left(c_{l_{1}}=0\right)$ case, since then the cofactor matrix of the phase data only appears in factor $f_{1}$. The graph shows that the ADOP is at a small value: even with $c_{\phi}=1 \mathrm{~cm}$ it is below 0.10 cyc. Also it can be seen that cross correlation lowers the ADOP, but this effect is marginal.

Concerning the code data, they only play a role for the geometry-fixed ADOP in case the ionospheric delays are estimated (since their cofactor matrix $C_{p}$ only shows up in the scalar I). Hence, the geometry-fixed ADOP as function of the code precision is computed for the ionosphere-float $\left(c_{l_{1}}=\infty\right)$ case in Fig. 3 (top middle; again for a single epoch based on six satellites and no satellite-dependent weights). In this graph also the effect of cross correlation between code observables on two frequencies can be seen, assuming a similar cofactor matrix as in Eq. (26), but now for code data. Again correlation coefficients of $c=0, c=0.5$ and $c=0.75$ were used and, as can be seen in the figure, code cross correlation has a negative effect on ADOP, although it is slight. It can also be seen that with $c_{p}=30 \mathrm{~cm}$ the ADOP is about 0.25 cyc, which is too large for successful ambiguity resolution. However, with $c_{p}=5 \mathrm{~cm}$, the ADOP has decreased to about 0.10 cyc. Thus, if - in the light of GPS modernizationthe code precision will improve, instantaneous ambiguity resolution in the geometry-fixed ionosphere-float case might become feasible.

\subsection{Ionospheric delay weighting and number of frequencies}

The large ADOP values in the ionosphere-float case may become significantly smaller if the ionospheric delays are weighted in the model. In absence of phase and code cross correlation, the ionosphere factor becomes (in the dualfrequency case) $I=\xi+\kappa /\left(\mu_{1}^{2}+\mu_{2}^{2}\right)$, with $\xi=c_{\phi}^{2} / c_{p}^{2}$, i.e., the phase-code variance ratio, and $\kappa=c_{\phi}^{2} / c_{l_{1}}^{2}$, i.e., the phase-ionosphere variance ratio. In case of GPS, assuming $c_{\phi}=3 \mathrm{~mm}, c_{p}=30 \mathrm{~cm}$, factor $f_{4}$ is approximately $f_{4} \simeq 10$, but in the ionosphere-weighted case, assuming $c_{l_{1}}=1 \mathrm{~cm}$, it is reduced to $f_{4} \simeq 2.5$, thus about a factor 4 better for ADOP. Figure 3 (top right) shows the dual-frequency ADOP as function of the ionospheric standard deviation. It is clearly an S-curve: for small values of $c_{l_{1}}$ the ionospherefixed ADOP is approximated, while for large values the ionosphere-weighted ADOP approximates its ionospherefloat counterpart. In the latter case the ionospheric information does not contribute at all to ADOP.

Instead of ionospheric weighting, the ionosphere-float ADOP is lowered when more than two frequencies are available. Again with the assumptions concerning the precision of the GPS phase and code data as above, it follows that in the ionosphere-float case $I=\xi$ and thus $f_{4} \simeq 10^{2 / j}$. This approximation shows the beneficial effect of the number of frequencies: while with just one frequency $f_{4}$ is about a factor 100 , with two frequencies this factor is already reduced to 10 . With three frequencies it will be reduced to about 4.6. In Fig. 3 (bottom left), the ionosphere-float ADOP is shown 
as function of the number of frequencies. From this graph it follows that with a modernized triple-frequency GPS, instantaneous ambiguity resolution may become feasible for longer baselines for which it is not needed to estimate coordinates. Note that in the same graph we also plotted a value for $j=4$ frequencies, to show the effect of this additional frequency. The wavelength of this fourth signal has been taken equal to the GPS L5 signal.

\subsection{Time correlation and number of samples}

In the previous subsections we computed instantaneous ADOPs, thus based on a single epoch of data. In that case, $f_{2}=1$. In this subsection we study the impact of increasing the number of samples. If these samples are uncorrelated in time, $R_{k}=I_{k}$, and $f_{2}=\sqrt{1 / k}$. If there are temporal correlations, and these can for example be modeled by a first-order autoregressive stochastic process, matrix $R_{k}$ in factor $f_{2}$ then becomes, see e.g., Priestley (1981):

$$
R_{k}=\left[\begin{array}{llll}
1 & \beta & \ldots & \beta^{k-1} \\
\beta & 1 & \ldots & \beta^{k-2} \\
\vdots & \vdots & \ddots & \vdots \\
\beta^{k-1} & \beta^{k-2} & \ldots & 1
\end{array}\right], \quad 0 \leq \beta<1
$$

It can be shown that in this case factor $f_{2}$ becomes

$$
f_{2}=\sqrt{\frac{1+\beta}{k-(k-2) \beta}}
$$

from which follows that in absence of time correlation, $\beta=$ $0, f_{2}=\sqrt{1 / k}$, but with full time correlation, i.e., $\beta=1$, the factor reduces to 1 , implying that the ADOP is not improved when the number of samples is increased. This is due to the fact that with large time correlation each subsequent sample provides less information to improve ambiguity resolution. Figure 3 (bottom middle) shows the ADOP vs. number of samples for $\beta=0, \beta=0.5$ and $\beta=1$.

\subsection{Satellite-dependent weighting and number of satellites}

All previous graphs were based on the assumption that the weights of all satellites in matrix $W_{m}$ are set to 1 , i.e., $w_{s}=1$ for $s=1, \ldots, m$, from which follows that $f_{3}=m^{\frac{1}{2(m-1)}}$. Since the number of satellites lies in the range $m \in[2, \infty)$, it follows that $f_{3} \in[1, \sqrt{2}$ ), i.e., an increase of the number of satellites has only a marginal improvement on the geometryfixed ADOP. In GPS practice the accuracy of measurements often depends on the elevation under which the satellites are tracked. To account for these weights, we may for example use an exponential function cf. Euler and Goad (1991):

$$
w_{s}=1 /\left(1+\alpha \exp \left\{-\frac{\varepsilon^{s}}{\varepsilon^{8}}\right\}\right)^{2}, \quad s=1, \ldots, m
$$

Here $\varepsilon^{s}$ denotes the elevation of satellite $s, \varepsilon^{0}$ some reference elevation (usually the cut-off elevation) and $\alpha \geq 0$ a constant. We have assumed here that the elevations of the two receivers of the single baseline to the same satellite are approximately equal, such that $\varepsilon_{1}^{s} \simeq \varepsilon_{r}^{s} \doteq \varepsilon^{s}$ (this approximation is allowed for baselines with a length up to few hundred km). In Fig. 3 (bottom right), the geometry-fixed ADOP is plotted as function of the number of satellites for the values $\alpha=0, \alpha=2$ and $\alpha=4$. For this an instantaneous GPS receiver-satellite geometry was used, based on 10 satellites. The reference elevation is taken as $\varepsilon^{0}=15^{\circ}$. For the case $m=4$ the four satellites with the highest elevations were used, and from the remaining set of satellites the satellite with the highest elevation was selected for $m=5$. This procedure was repeated to compute the ADOPs up to $m=10$. Note from the figure that only in absence of satellite-dependent weighting $(\alpha=0)$ the increase of number of satellites is (slightly) beneficial for ADOP. In both cases when $\alpha=2$ and $\alpha=4$, the ADOPs even get larger for increasing $m$. This can be explained from the fact that the satellite which is added has a lower elevation than the satellites already included in the model. Consequently, the weight for the new satellite is relatively low, which implies that the new sum of the satellite weights, i.e., $\sum_{s=1}^{m} w_{s}$, will only be slightly higher than without the new satellite, however the product of weights, i.e., $\prod_{s=1}^{m} w_{s}$, will be much smaller than in the situation without the satellite. This then results in a much larger ratio of the sum and product as in factor $f_{3}$ in the case with the new satellite, and this may lead to a larger factor $f_{3}$, despite that the power $1 / 2(m-1)$ increases as due to the additional satellite. As consequence, the ADOP will get larger in the new situation.

\section{Geometry-free ADOP expression}

To derive closed-form expressions for the ADOP of the geometry-free model, we again use the determinantal relation in Eq. (17). For the geometry-free model the determinant of the ambiguity vc-matrix, denoted as $Q_{\hat{a}}^{\mathrm{GFr}}$, can then be evaluated as:

$\left|Q_{\hat{a}}^{\mathrm{GFr}}\right|=\left|Q_{\hat{a} \mid \rho}\right| \frac{\left|Q_{\hat{\rho}}\right|}{\left|Q_{\check{\rho}}\right|}$

where $Q_{\hat{\rho}}$ and $Q_{\breve{\rho}}$ denote the vc-matrices of the ambiguityfloat and ambiguity-fixed DD range parameters, respectively, and $Q_{\hat{a} \mid \rho}$ the ambiguity vc-matrix of the geometry-fixed model. Hence, the first part of the geometry-free ADOP expression will be formed by the geometry-fixed ADOP in Eq. (25). For the second part of the expression we need analytical expressions for the float and fixed vc-matrices of the range parameters. It can be shown (see MGP (2007)) that 
these are given as:

$$
\begin{aligned}
& Q_{\hat{\rho}}=2 c_{\check{\rho}}^{2}\left[R_{k}+\left(\frac{c_{\hat{\rho}}^{2}}{c_{\check{\rho}}^{2}}-1\right) \frac{1}{\left[e_{k}^{T} R_{k}^{-1} e_{k}\right]} e_{k} e_{k}^{T}\right] \otimes D_{m}^{T} W_{m}^{-1} D_{m} \\
& Q_{\check{\rho}}=2 c_{\check{\rho}}^{2} R_{k} \otimes D_{m}^{T} W_{m}^{-1} D_{m}
\end{aligned}
$$

In these expressions the variance factors of the float and fixed ranges are computed as:

$$
\begin{aligned}
c_{\hat{\rho}}^{2} & =\frac{\left[\mu^{T} C_{p}^{-1} \mu\right]+c_{l_{1}}^{-2}}{\left[e_{j}^{T} C_{p}^{-1} e_{j}\right]\left(\left[\mu^{T} C_{p}^{-1} \mu\right]+c_{l_{1}}^{-2}\right)-\left[e_{j}^{T} C_{p}^{-1} \mu\right]^{2}} \\
c_{\grave{\rho}}^{2} & =\frac{\left[\mu^{T}\left(C_{\phi}^{-1}+C_{p}^{-1}\right) \mu\right]+c_{l_{1}}^{-2}}{\left[e_{j}^{T}\left(C_{\phi}^{-1}+C_{p}^{-1}\right) e_{j}\right]\left(\left[\mu^{T}\left(C_{\phi}^{-1}+C_{p}^{-1}\right) \mu\right]+c_{l_{1}}^{-2}\right)-\left[e_{j}^{T}\left(C_{\phi}^{-1}-C_{p}^{-1}\right) \mu\right]^{2}}
\end{aligned}
$$

Note the similarities in the expressions for $Q_{\hat{\rho}}$ and $Q_{\check{\rho}}$ on the one hand, and the expressions for $Q_{\hat{\imath}_{1} \mid \rho}$ and $Q_{\breve{l}_{1} \mid \rho}$ in Eq. (20) on the other hand: the structure of the float and fixed expressions are exactly the same, except that in Eq. (31) ' $\rho$ ' replaces ' $l_{1} \mid \rho$ ' in Eq. (20). Consequently, the determinant ratio of the float and fixed range vc-matrices has a similar structure as that of the float and fixed ionosphere vc-matrices conditioned on the ranges, see Eq. (23):

$\frac{\left|Q_{\hat{\rho}}\right|}{\left|Q_{\check{\rho}}\right|}=\left[\frac{c_{\hat{\rho}}^{2}}{c_{\check{\rho}}^{2}}\right]^{m-1}$

In a similar manner as the ratio of the ionospheric variance factors, we may rewrite the ratio of float and fixed range variance factors as:

$$
\frac{c_{\hat{\rho}}^{2}}{c_{\check{\rho}}^{2}}=1+\frac{1}{\delta}
$$

where scalar $\delta$ is referred to as range factor. Note that in the ionosphere-fixed case $\left(c_{l_{1}}=0\right)$, this range factor reduces to $\delta=\left[e_{j}^{T} C_{p}^{-1} e_{j}\right] /\left[e_{j}^{T} C_{\phi}^{-1} e_{j}\right]$.

Thus, the geometry-free ADOP expression is derived as

$$
\mathrm{ADOP}^{\mathrm{GFr}}=\mathrm{ADOP}^{\mathrm{GFi}} \underbrace{\left[1+\frac{1}{\delta}\right]^{\frac{1}{2 j}}}_{f_{5}}
$$

In case we only have phase measurements available, $C_{p}^{-1}=$ 0 , the denominator of the upper ratio in Eq. (32) becomes zero, which corresponds to $c_{\hat{\rho}}^{2}=\infty$. Equivalently, in that case $\delta=0$, such that ADOP goes to infinity. This is explained from the fact that the geometry-free model is not solvable in absence of code data (see Table 5).

\subsection{Simplification of float and fixed range variance factors}

The expressions for the float and fixed variance factors of the ranges are quite complex, see Eq. (32). They can be simplified when it is assumed that the phase and code data have equal standard deviations on different frequencies and there is no cross correlation between them. In that case, the phase and code cofactor matrices reduce to scaled unit matrices, i.e., $C_{\phi}=c_{\phi}^{2} I_{j}$ and $C_{p}=c_{p}^{2} I_{j}$. The float and fixed range variance factors then can be written as

$$
\begin{aligned}
& c_{\hat{\rho}}^{2}=\frac{c_{p}^{2}}{j} \frac{\left[\mu^{T} \mu\right]+\kappa / \xi}{(\mu-\bar{\mu})^{T}(\mu-\bar{\mu})+\kappa / \xi} \\
& c_{\check{\rho}}^{2}=\frac{c_{\phi}^{2}}{j(1+\xi)} \frac{\left[\mu^{T} \mu\right]+\kappa /(1+\xi)}{(\mu-\bar{\mu})^{T}(\mu-\bar{\mu})+\kappa /(1+\xi)+\frac{4 \xi}{j(1+\xi)^{2}}\left[e_{j}^{T} \mu\right]^{2}}
\end{aligned}
$$

with again $\xi$ the phase-code variance ratio and $\kappa$ the phaseionosphere variance ratio. Moreover, $(\mu-\bar{\mu})^{T}(\mu-\bar{\mu})=\left[\mu^{T} \mu\right]-$ $\frac{1}{j}\left[e_{j}^{T} \mu\right]^{2}$, with $\bar{\mu}=\frac{1}{j}\left[e_{j}^{T} \mu\right] e_{j}$, i.e., a $j \times 1$ vector with the average of the entries of $\mu$ at all its entries. We now consider these variance factors in the ionosphere-fixed and ionosphere-float cases. In the ionosphere-fixed case, $\kappa=\infty$ and consequently $c_{\hat{\rho} \mid l_{1}}^{2}=c_{p}^{2} \frac{1}{j}$ and $c_{\check{\rho} \mid l_{1}}^{2}=c_{\phi}^{2} \frac{1}{j(1+\xi)}$. Thus, $c_{\hat{\rho} \mid l_{1}}^{2} / c_{\check{\rho} \mid l_{1}}^{2}=$ $1+1 / \xi$, which implies that $\delta=\xi$, i.e., the phase-code variance ratio. In the ionosphere-float case, $\kappa=0$, and it can be proved that for the float-fixed variance ratio of the ranges the following expression holds:

$\frac{c_{\hat{\rho}}^{2}}{c_{\check{\rho}}^{2}}=\left(1+\frac{1}{\xi}\right)+\frac{4}{j(1+\xi)} \frac{\left[e_{j}^{T} \mu\right]^{2}}{(\mu-\bar{\mu})^{T}(\mu-\bar{\mu})}$

It can be shown that for current GPS the second term on the right hand side of Eq. (37) is significantly smaller than the first term (about $10^{4}$ ), and may be neglected. From this the conclusion follows that in both ionosphere-fixed and -float cases the scalar $\delta$ is (approximately) equal to the phase-code variance ratio $\xi$. Consequently, the parametrization of the receiversatellite ranges has the following worsening effect on ADOP: compared with the geometry-fixed ADOP the geometry-free ADOP is a factor 100 larger in the single-frequency case, while in the dual-frequency case the geometry-free ADOP is ten times its geometry-fixed counterpart. An exception to this is the single-frequency ionosphere-float case. If $j=1$, it follows that $\mu=\bar{\mu}$ and if also $\kappa=0$, the denominator of the expression for the float range variance factor reduces to zero (see Eq. (36)) and thus $c_{\hat{\rho}}^{2}=\infty$, which implies that $\delta=0$. This is of course due to the fact that at least two frequencies are needed when the ionospheric delays are assumed as complete unknowns in the geometry-free model.

Similar as in Fig. 3 for the geometry-fixed model, by means of Fig. 4 the sensitivity of the geometry-free ADOP to changes in the model is given. In the upper three graphs the 
Fig. 4 Sensitivity of geometry-free ADOPs to changes in the model. For the three graphs at top it holds that $k=1, j=2$ and $m=6$, while for the three graphs at bottom it holds that $c_{\phi}=0.003 \mathrm{~m}, c_{p}=$ $0.30 \mathrm{~m}$ and $c_{l_{1}}=0$
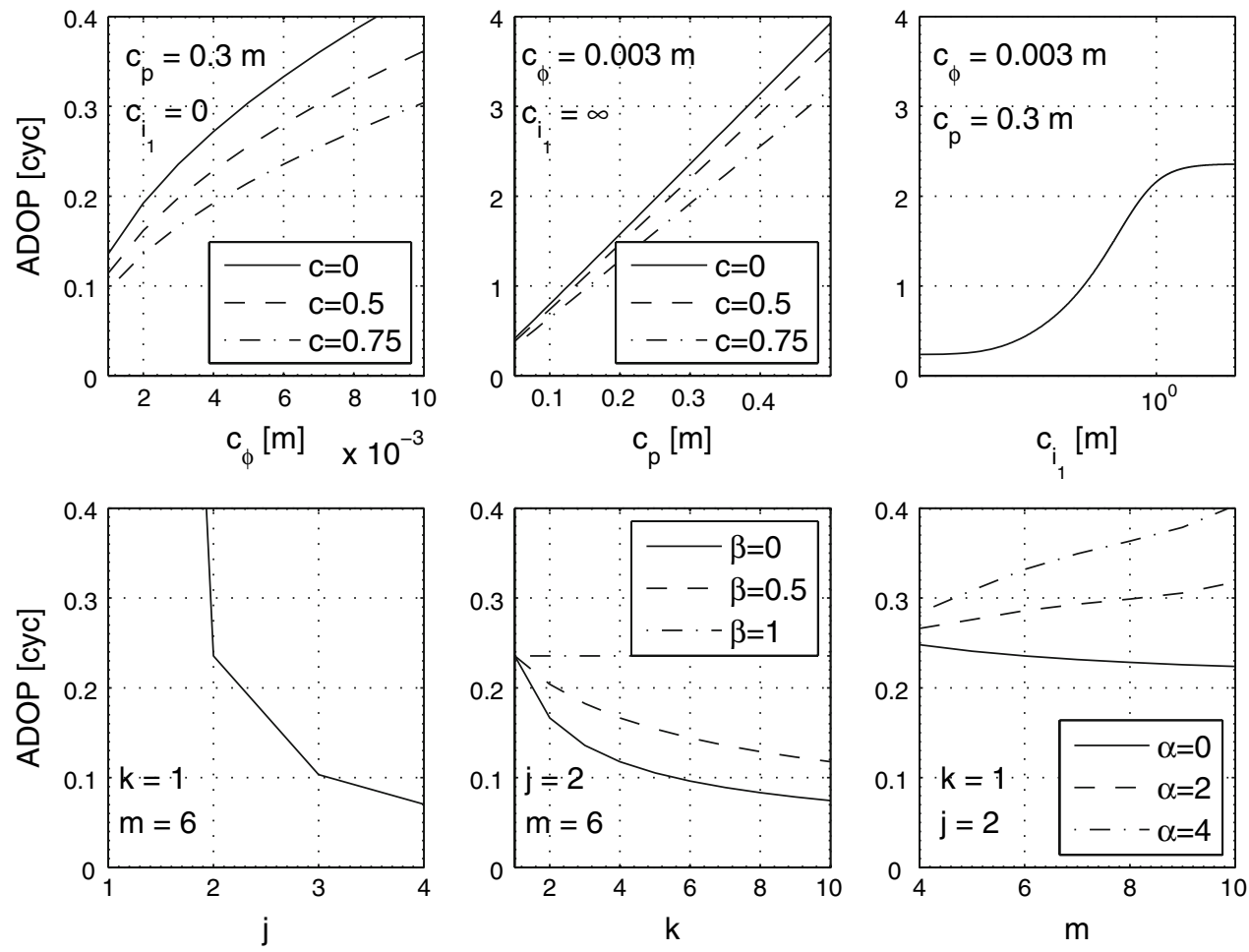

sensitivity of ADOP with respect to changes in cofactor matrices of phase, code and ionosphere observations, i.e., $C_{\phi}, C_{p}$ and $c_{l_{1}}^{2}$ can be inferred. Compared to the graphs of the geometry-fixed model, one immediately may notice the worsening effect of the parametrization of the receiversatellite ranges. Comparing for example the graph at top right with its geometry-fixed counterpart in Fig. 3, the factor 10 (as derived above) for the ionosphere-fixed and ionosphere-float cases can be easily seen. The three lower graphs in Fig. 4, in which the number of frequencies, number of epochs and number of satellites are varied, are exactly the same as those at bottom of Fig. 3. However the three graphs of the geometryfree model concern the ionosphere-float case, while those of the geometry-fixed model refer to the ionosphere-fixed case. Obviously, the parametrization of the DD ranges (and keeping the DD ionospheric delays fixed) has the same net effect on ADOP as the parametrization of DD ionospheric delays (and keeping the DD ranges fixed) in the geometry-fixed model.

\section{Geometry-based ADOP expressions}

In analogous manner as the geometry-free model, the determinant of the ambiguity vc-matrix of any of the three geometry-based models, denoted as $Q_{\hat{a}}^{\mathrm{GB}}$, can be given as:

$\left|Q_{\hat{a}}^{\mathrm{GB}}\right|=\left|Q_{\hat{a} \mid g}\right| \frac{\left|Q_{\hat{g}}\right|}{\left|Q_{\check{g}}\right|}$ with $Q_{\hat{a} \mid g}=Q_{\hat{a} \mid \rho}$ the ambiguity vc-matrix of the geometryfixed model and where $Q_{\hat{g}}$ and $Q_{\breve{g}}$ denote the vc-matrices of the ambiguity-float and ambiguity-fixed baseline parameters, respectively. Consequently, the first part of the geometrybased ADOP expression is-like in the geometry-free caseformed by the geometry-fixed ADOP in Eq. (25). In the following subsections closed-form expressions are derived for the ratios of the determinants of float and fixed baseline vc-matrices for the three different versions of the geometrybased model.

\subsection{Moving receiver, short time span}

Closed-form expressions for the float and fixed baseline vcmatrices of the moving-receiver short-time geometry-based model can be shown to be, see MGP (2007):

$$
\begin{aligned}
& Q_{\hat{g}}=2 c_{\check{\rho}}^{2}\left[R_{k}+\frac{1}{\delta} \frac{1}{\left[e_{k}^{T} R_{k}^{-1} e_{k}\right]} e_{k} e_{k}^{T}\right] \otimes\left[\bar{G}^{T} P_{m} \bar{G}\right]^{-1} \\
& Q_{\check{g}}=2 c_{\check{\rho}}^{2} R_{k} \otimes\left[\bar{G}^{T} P_{m} \bar{G}\right]^{-1}
\end{aligned}
$$

where $g=\left[g(1)^{T}, \ldots, g(k)^{T}\right]^{T}$. Note the similarities of these expressions with those of the geometry-free model, see Eq. (31). The satellite-dependent projector $P_{m}$ reads as:

$P_{m}=W_{m}-\frac{1}{e_{m}^{T} W_{m} e_{m}} W_{m} e_{m} e_{m}^{T} W_{m}$

Since this projector is invariant to the choice of pivot satellite, the baseline vc-matrices are invariant as well. For the 
evaluation of the determinant ratio of both, note that the parts of the vc-matrices at the left side of the Kronecker symbol in Eq. (39) are exactly the same as those at the left side in Eq. (31), i.e., the vc-matrices of the geometry-free model. The parts at the right side of the Kronecker symbol are however different. But since these are the same in the float and fixed cases, the ratio of the determinants of the baseline vcmatrices is only governed by the dimension $v$ of the matrix at the right side of the Kronecker symbol. This dimension shows up as the power of the float-fixed range variance ratio, and thus, see Eq. (33):

$\frac{\left|Q_{\hat{g}}\right|}{\left|Q_{\breve{g}}\right|}=\left[\frac{c_{\hat{\rho}}^{2}}{c_{\check{\rho}}^{2}}\right]^{v}=\left[1+\frac{1}{\delta}\right]^{v}$

Consequently, the ADOP expression follows as:

$$
\mathrm{ADOP}^{\mathrm{MR}-\mathrm{ST}}=\mathrm{ADOP}^{\mathrm{GFi}} \underbrace{\left[1+\frac{1}{\delta}\right]^{\frac{v}{2 j(m-1)}}}_{f_{5}}
$$

This result will be discussed in the next subsection after deriving the stationary-receiver short-time ADOP.

\subsection{Stationary receiver, short time span}

For both stationary receivers during the (short) time span, the following baseline vc-matrices are derived:

$$
\begin{aligned}
& Q_{\hat{g}}=2 c_{\hat{\rho}}^{2} \frac{1}{\left[e_{k}^{T} R_{k}^{-1} e_{k}\right]}\left[\bar{G}^{T} P_{m} \bar{G}\right]^{-1} \\
& Q_{\check{g}}=2 c_{\check{\rho}}^{2} \frac{1}{\left[e_{k}^{T} R_{k}^{-1} e_{k}\right]}\left[\bar{G}^{T} P_{m} \bar{G}\right]^{-1}
\end{aligned}
$$

For a proof we again refer to MGP (2007). Note that both float and fixed expressions in Eq. (43) only differ in their range variance factors. For the determinant ratio of both vcmatrices therefore only these variance factors remain, which implies that the determinant ratio is exactly equal to the ratio of the moving-receiver model, see Eq. (41). So now we have the important result that although the individual baseline vc-matrices of the moving-receiver and stationary-receiver models differ, the gain in baseline precision due to ambiguity fixing of both models is the same, thus irrespective whether the second receiver is moving or not. As consequence, the ADOP expressions of both models are exactly the same:

$$
\mathrm{ADOP}^{\mathrm{SR}-\mathrm{ST}}=\mathrm{ADOP}^{\mathrm{MR}-\mathrm{ST}}
$$

Comparing factor $f_{5}$ in Eq. (42) reveals that is very similar to its geometry-free counterpart, see Eq. (35): the difference lies in the powers of factor $f_{5}$. For $m=v+1$, i.e., the minimum number of satellites in the geometry-based models, both geometry-based and geometry-free ADOPs even become equivalent, but for $m>v+1$ however, the geometry-based ADOPs start to become significantly smaller than their geometry-free counterparts. For example, with $v=3$ and $m=7$, the geometry-based factor becomes $f_{5}=\sqrt{100}=$ 10 for single-frequency data and $f_{5}=\sqrt{10} \simeq 3$ for dualfrequency data, while for the geometry-free model these factors are 100 and 10, respectively. This illustrates the beneficial contribution of the number of satellites in the geometrybased model. This improvement is also visible when the graphs in Fig. 5 are compared with their geometry-free counterparts in Fig. 4. Comparing the graphs at bottom right, it can be seen that in case of the geometry-based model an increase of the number of satellites is always beneficial, while in case of the geometry-free model this also depends on the way the observations are weighted in the stochastic model. In presence of an elevation-dependent weighting, an increase of the number of satellites may even lead to an increase of ADOP in case of the geometry-free model, however not in case of the geometry-based model, which is due to the more beneficial effect of the number of satellites on factor $f_{5}$, rather than on factor $f_{3}$ in which the elevation-dependent weighting shows up.

\subsubsection{Example: Instantaneous geometry-based ADOPs}

As an illustration, we computed ADOPs based on the receiver-satellite geometry at permanent GPS station Delft $\left(52.0^{\circ} \mathrm{N}, 4.4^{\circ} \mathrm{E}\right)$, the Netherlands, for the day 1 January 2003 (30s sampling interval; cut-off elevation: $15^{\circ}$ ). Singleepoch ADOPs have been computed based on dual-frequency GPS data, using the assumptions of uncorrelated phase and code data, with $c_{\phi}=0.003 \mathrm{~m}$ and $c_{p}=0.30 \mathrm{~m}$. No satellitedependent weights have been applied and tropospheric zenith delays are not parameterized $(v=3)$. The ionospheric standard deviation was set to $c_{l_{1}}=1 \mathrm{~cm}$. If we assume a baselinelength dependent function of $c_{l_{1}}=a \cdot l_{1 r}$, with $a=$ $0.68 \mathrm{~mm} / \mathrm{km}$ cf. Schaffrin and Bock (1988), then this corresponds to a baseline of about $15 \mathrm{~km}$. Fig. 6 shows the ADOPs for the 2880 epochs. The figure also shows the ADOP-based ambiguity success rates $P_{\mathrm{ADOP}}$ (see Eq. (13)) during the day. As can be seen from this graph is that this success rate approaches 1 for most times of the day, except when there are five or less satellites available.

\subsection{Stationary receiver, long time span}

For the long-time geometry-based model, the float and fixed vc-matrices of the baseline components are derived as, see MGP (2007):

$$
\begin{aligned}
Q_{\hat{g}}= & 2 c_{\check{\rho}}^{2}\left[\frac{\delta}{\delta+1} \sum_{i=1}^{k} G(i)^{T} P_{m} G(i)\right. \\
& \left.+\frac{1}{\delta+1} \sum_{i=1}^{k}(G(i)-\bar{G})^{T} P_{m}(G(i)-\bar{G})\right]^{-1} \\
Q_{\breve{g}}= & 2 c_{\check{\rho}}^{2}\left[\sum_{i=1}^{k} G(i)^{T} P_{m} G(i)\right]^{-1}
\end{aligned}
$$


Fig. 5 Sensitivity of the short-time geometry-based ADOPs to changes in the model. For the three graphs at top it holds that $k=1, j=2$ and $m=6$, while for the three graphs at bottom it holds that $c_{\phi}=0.003 \mathrm{~m}, c_{p}=0.30 \mathrm{~m}$ and $c_{l_{1}}=0$. In all graphs $v=3$

Fig. 6 ADOP, $P_{\mathrm{ADOP}}$ and number of satellites vs. observation epochs, for dual-frequency ionosphere-weighted GPS, with $c_{l_{1}}=1 \mathrm{~cm}$
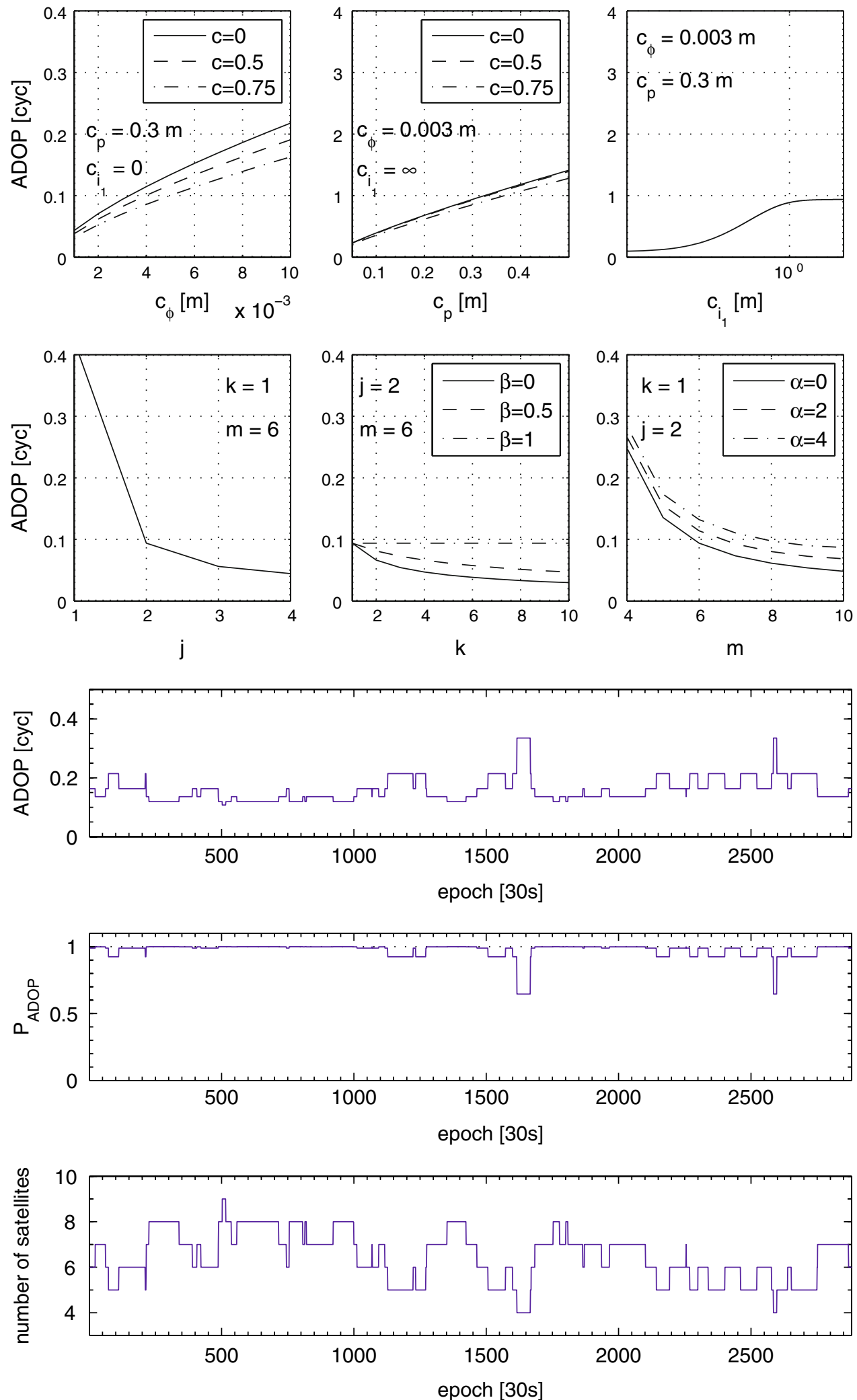

where the projector $P_{m}$ is obtained from Eq. (40) with $W_{m}=I_{m}$, so $P_{m}=I_{m}-\frac{1}{m} e_{m} e_{m}^{T}$. Note that if all individual time-varying geometry matrices $G(i)$ are replaced by their time-averaged counterpart, i.e., $\bar{G}$, the baseline vc-matrices in Eq. (45) reduce to their counterparts of the stationary-receiver short-time model, see Eq. (43) 
(using the property that $c_{\tilde{\rho}}^{2} \frac{\delta+1}{\delta}=c_{\hat{\rho}}^{2}$, and with $R_{k}$ $=I_{k}$ ).

In order to derive a closed-form expression for the ratio of the determinants of the float and fixed vc-matrices, first consider the baseline vc-matrices in case we only have phase data available. Setting $\delta=0$ in Eqs. (45) results in:

$$
\begin{aligned}
& Q_{\hat{g}}(\phi)=2 c_{\check{\rho}}^{2}(\phi)\left[\sum_{i=1}^{k}(G(i)-\bar{G})^{T} P_{m}(G(i)-\bar{G})\right]^{-1} \\
& Q_{\breve{g}}(\phi)=2 c_{\check{\rho}}^{2}(\phi)\left[\sum_{i=1}^{k} G(i)^{T} P_{m} G(i)\right]^{-1}
\end{aligned}
$$

with $Q_{\hat{g}}(\phi)$ and $Q_{\breve{g}}(\phi)$ the float and fixed baseline vcmatrices based on phase data only and where $c_{\breve{\rho}}^{2}(\phi)$ denotes the phase-only variance factor of the fixed ranges, which can be computed from the second ratio in Eq. (32) by setting $C_{p}^{-1}=0$. When the determinant ratio of these phase-only vc-matrices is taken, it easily follows that the variance factor $c_{\check{\rho}}^{2}(\phi)$ gets eliminated and that the result only depends on the matrices $G(i), \bar{G}$ and $P_{m}$. This means that in the phaseonly case the determinant ratio does not depend on the a priori assumptions in the stochastic model, but only on the length of the observation time span, the number of satellites and their relative geometry with respect to the receiver. In Teunissen (1997a) it was shown that the expressions for the phase-only baseline vc-matrices can be rewritten using the following canonical decomposition:

$$
\begin{aligned}
& Q_{\hat{g}}(\phi)=2 c_{\check{\rho}}^{2}(\phi)\left[F \Gamma^{-1} F^{T}\right]^{-1} \\
& Q_{\breve{g}}(\phi)=2 c_{\check{\rho}}^{2}(\phi)\left[F F^{T}\right]^{-1}
\end{aligned}
$$

with $\Gamma=\operatorname{diag}\left(\gamma_{1}, \ldots, \gamma_{v}\right)$, and where $\gamma_{i}, i=1, \ldots, v$ denote the roots of the following characteristic equation:

$\left|Q_{\hat{g}}(\phi)-\gamma Q_{\breve{g}}(\phi)\right|=0$

These $v$ eigenvalues measure the gain in baseline precision due to ambiguity fixing and will therefore be referred to as baseline gain numbers. Matrix $F=\left(\tau_{1}, \ldots, \tau_{v}\right)$ contains the eigenvectors corresponding to these gain numbers, and are consequently referred to as gain vectors, for which thus holds that $Q_{\hat{g}}(\phi) F=Q_{\breve{g}}(\phi) F \Gamma$. These gain numbers are completely governed by the change of the receiver-satellite geometry: if there is no change (in case of a single epoch), the gain numbers are infinitely large, while with an enormous change of the geometry they approximate unity. Hence the gain numbers range within the interval $\gamma_{i} \in[1, \infty)$, for $i=$ $1, \ldots, v$. Using the canonical decomposition, the phase-only determinant ratio can now be written as:

$$
\frac{\left|Q_{\hat{g}}(\phi)\right|}{\left|Q_{\breve{g}}(\phi)\right|}=\frac{\left|F F^{T}\right|}{\left|F \Gamma^{-1} F^{T}\right|}=\frac{|F|\left|F^{T}\right|}{|F|\left|\Gamma^{-1}\right|\left|F^{T}\right|}=|\Gamma|=\prod_{i=1}^{v} \gamma_{i}
$$

Similar to Eqs. (47), a canonical decomposition for the general case can be given as:

$$
\begin{aligned}
Q_{\hat{g}} & =2 c_{\check{\rho}}^{2}\left[\frac{\delta}{\delta+1} F\left(I_{v}+\frac{1}{\delta} \Gamma^{-1}\right) F^{T}\right]^{-1} \\
Q_{\breve{g}} & =2 c_{\check{\rho}}^{2}\left[F F^{T}\right]^{-1}
\end{aligned}
$$

Their determinant ratio can then be written as:

$$
\frac{\left|Q_{\hat{g}}\right|}{\left|Q_{\grave{g}}\right|}=\frac{|F|\left|F^{T}\right|}{|F|\left|\frac{\delta}{\delta+1} I_{v}+\frac{1}{\delta+1} \Gamma^{-1}\right|\left|F^{T}\right|}=\frac{1}{\left|\frac{\delta}{\delta+1} I_{v}+\frac{1}{\delta+1} \Gamma^{-1}\right|}
$$

Since both matrices $I_{v}$ and $\Gamma^{-1}$ are diagonal, this ratio can even be more simplified as:

$\frac{\left|Q_{\hat{g}}\right|}{\left|Q_{\grave{g}}\right|}=\prod_{i=1}^{v} \frac{\delta+1}{\delta+1 / \gamma_{i}}=\prod_{i=1}^{v}\left(1+\frac{1-1 / \gamma_{i}}{\delta+1 / \gamma_{i}}\right)$

Note that in absence of code data, $\delta=0$, the determinant ratio above reduces to $\prod_{i=1}^{v} \gamma_{i}$, indeed corresponding to the phase-only ratio we found earlier, see Eq. (49).

Consequently, the ADOP expression for the stationaryreceiver long-time model can be given as:

$\mathrm{ADOP}^{\mathrm{SR}-\mathrm{LT}}=\mathrm{ADOP}^{\mathrm{GFi}}[\underbrace{\left[\prod_{i=1}^{v}\left(1+\frac{1-1 / \gamma_{i}}{\delta+1 / \gamma_{i}}\right)\right]^{\frac{1}{2 j(m-1)}}}_{f_{5}}$

Factor $f_{5}$ in Eq. (53) clearly shows the beneficial effect of a receiver-satellite geometry that is changing. If $\gamma_{i}=\infty$, $i=1, \ldots, v$, i.e., in case of a single observation epoch, the geometry does not change and factor $f_{5}$ reduces to its counterpart of the short-time geometry-based models, see Eq. (42). This is understandable, since the geometry in the short-time models does not change as well (they are based on the averaged receiver-satellite geometry over the time span). Increasing the time span implies that the gain numbers become smaller, and also factor $f_{5}$. In the case of an infinitely long time span, $\gamma_{i}=1, i=1, \ldots, v$, factor $f_{5}$ reduces to 1 , such that the long-time ADOP equals the geometry-fixed ADOP.

To demonstrate the effect of a changing receiver-satellite geometry on ADOP, we have again used the geometry of permanent GPS station Delft $\left(52.0^{\circ} \mathrm{N}, 4.4^{\circ} \mathrm{E}\right)$ in the Netherlands, during a 50-min observation time span on 1 January 2003 (data collected from 1.55 to 2.45 UTC, based on a 30 s sampling interval). Figure 7 gives the tracks of the satellites in an azimuth-elevation plot. For this receiver-satellite geometry we computed gain numbers, which are given as function of the time span in Fig. 8. These gain numbers have not only been computed based on a full available geometry using $m=8$ satellites (bottom graphs), but also based on a subset 


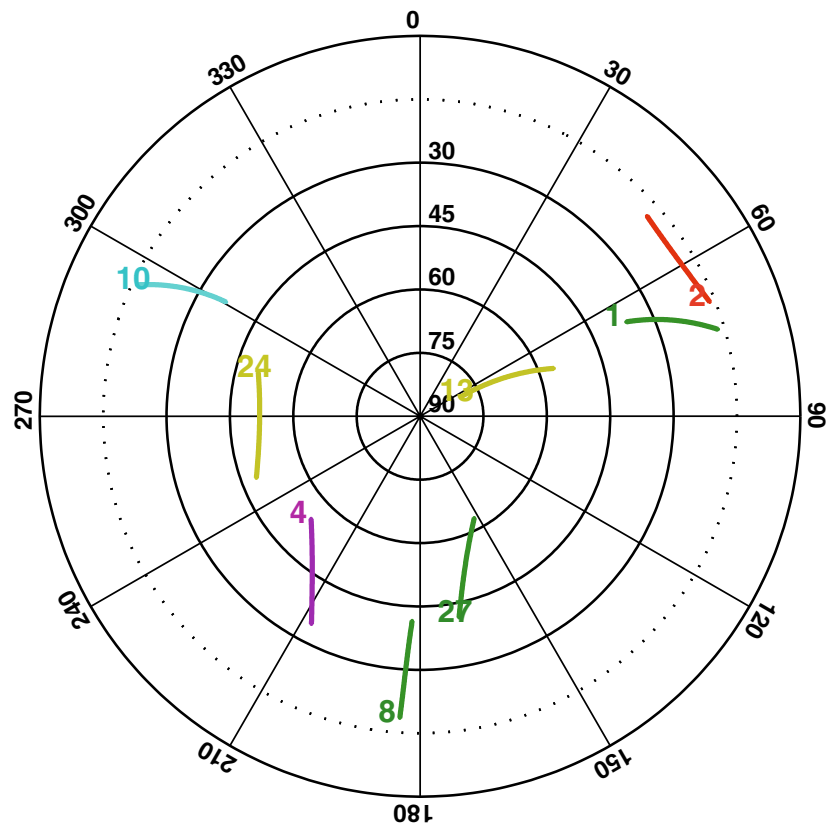

Fig. 7 GPS skyplot at Delft, the Netherlands during the observation time span 2.55-3.45 UTC on 1 January 2003 of $m=5$ satellites (top graphs). In case $m=5$, only PRNs 1 , 8, 10, 13 and 24 (see Fig. 7) are used. In addition, we distinguish between a scenario in which a tropospheric zenith delay is estimated while the receiver coordinates are held fixed ( $v=1$; left graphs), and also a scenario in which they are all estimated ( $v=4$; right graphs). The tropospheric mapping function is assumed as $\psi_{r}^{s}(i)=1 / \sin \varepsilon_{r}^{s}(i), s=1, \ldots, m$ with $\varepsilon_{r}^{s}(i)$ the elevation to satellite $s$. Figure 8 shows the decreasing gain numbers as function of the time span. The graphs also show that an increase of the number of satellites has some lowering impact on the gain numbers in case $v=4$, but not in case $v=1$. It can be seen that for five satellites the gain numbers in case $v=1$ are even (slightly) smaller than those for eight satellites. Based on the gain numbers in Fig. 8, in a next step the long-time ADOPs have been computed as function of the observation time span, based on the stochastic model assumptions, $C_{\phi}=c_{\phi}^{2} I_{j}, C_{p}=c_{p}^{2} I_{j}$, with $c_{\phi}=0.003 \mathrm{~m}$ and $c_{p}=0.30 \mathrm{~m}$. In addition, we used $c_{l_{1}}=\infty$, which means that the ADOPs are computed for the ionosphere-float case. The ADOPs are computed assuming dual-frequency data for $v=1$ and $v=4$, and for $v=4$ also based on three frequencies. To investigate the effect of the
Fig. 8 Gain numbers as function of observation time span, based on the receiver-satellite geometry as depicted in Fig. 7
Table 7 Observation time [min] needed for the ionosphere-float $\left(c_{l_{1}}=\infty\right)$ ADOP $<0.12$ cyc
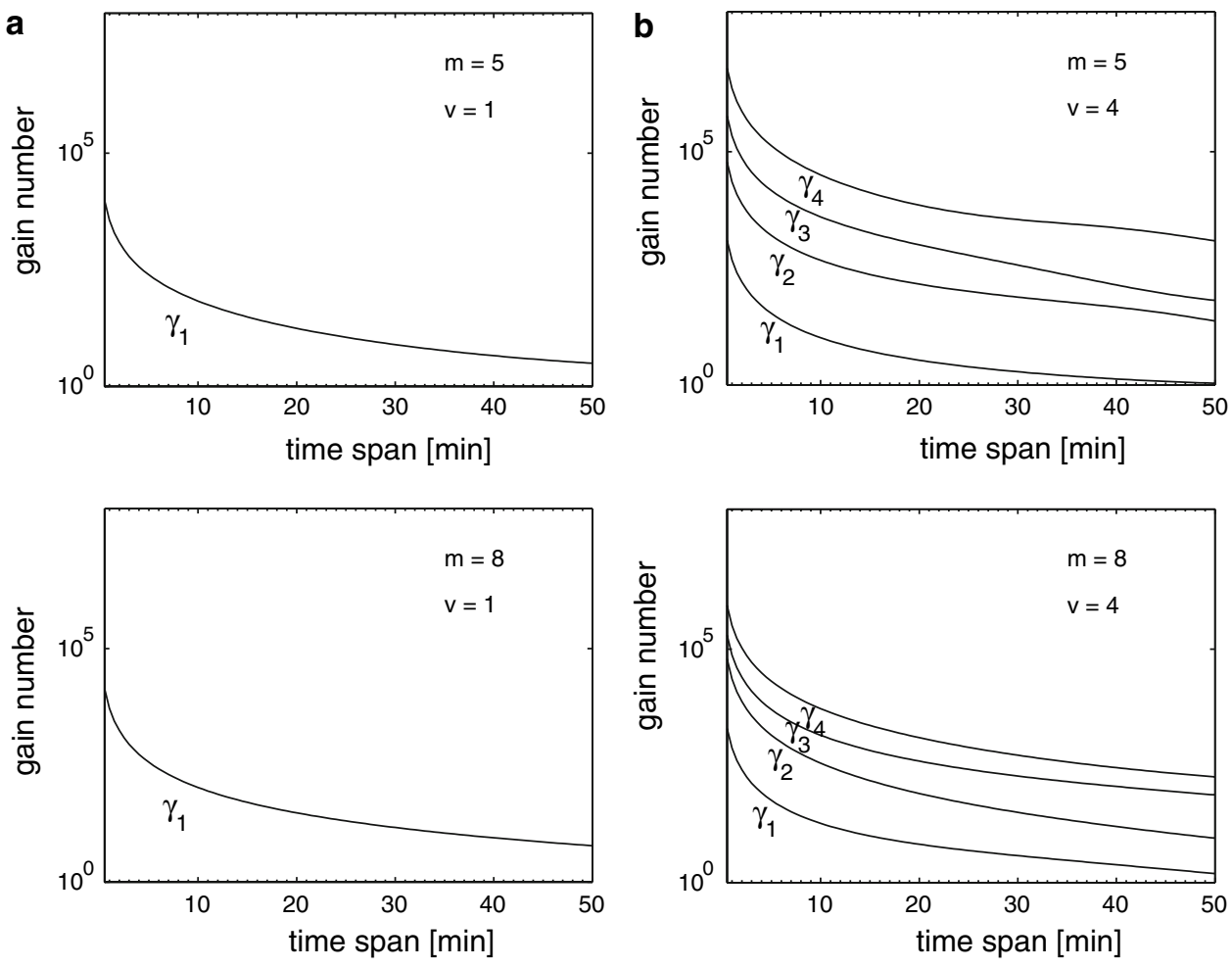

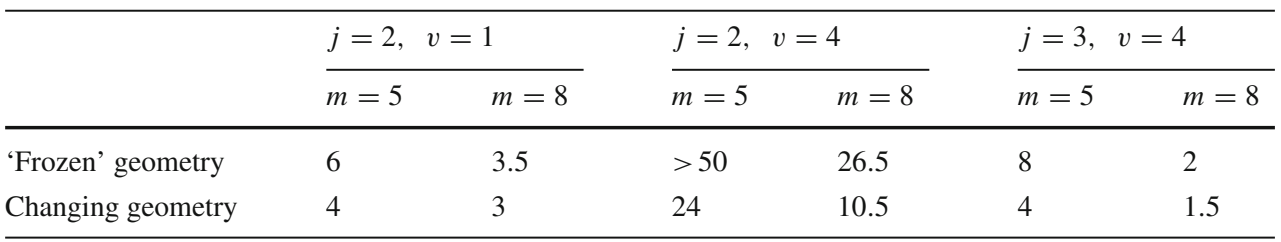


Table 8 Closed-form ADOP expressions as a product of 5 factors: ADOP $=f_{1} \times f_{2} \times f_{3} \times f_{4} \times f_{5}$ [cyc]

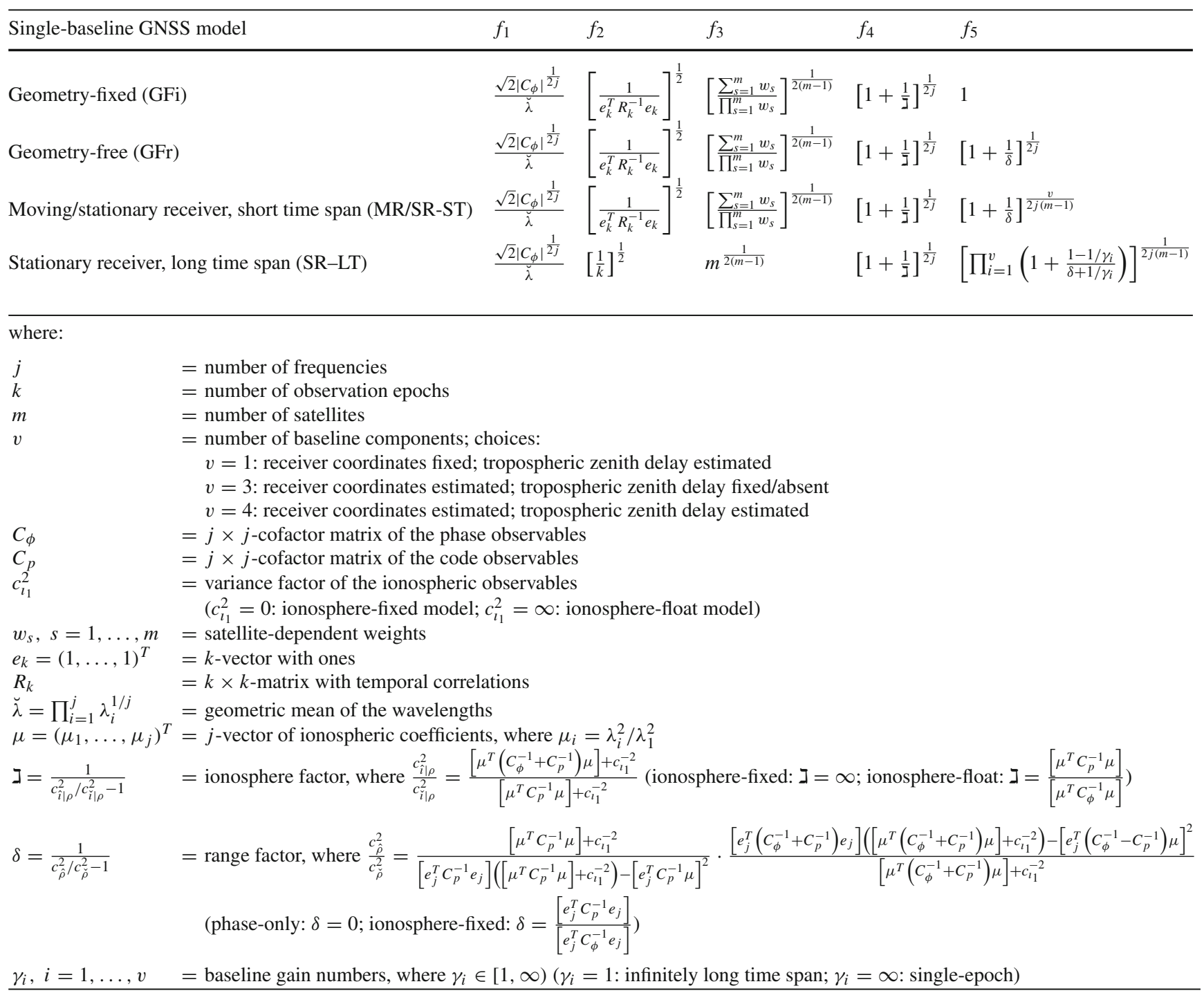

changing receiver-satellite geometry, the ADOPs have also been computed using a receiver-satellite geometry that does not change as function of the time span: a so called 'frozen' geometry, i.e., based on the geometry of the first epoch. The gain numbers based on this frozen geometry of course equal infinity. Table 7 presents values for the time span needed to obtain an ADOP smaller than 0.12 cyc, using the 'frozen' geometry as well as the changing geometry. From Table 7 it can be seen that based on a changing geometry the needed observation times are always shorter than based on a frozen geometry. Moreover, a changing receiver-satellite geometry is mainly beneficial in the dual-frequency case in which three coordinates and a troposphere parameter are estimated: compared to a frozen geometry, the observation time is more than halved to get the same level of ADOP. This effect is less pronounced for the dual-frequency case with only a tropospheric zenith delay and the triple-frequency case, since for those cases already with a frozen geometry relatively short time spans are feasible.

\section{Conclusions}

In this contribution we have presented closed-form analytical expressions for the ADOP. This ADOP is a relevant invariant measure for the success of GNSS ambiguity resolution, and is easy to compute. Closed-form ADOP expressions have been derived for a hierarchy of single-baseline GNSS models. These models include the geometry-fixed model, the geometry-free model and the geometry-based model, both for short and long observation time spans. The geometryfixed model is the strongest model and the geometry-free model is the weakest. Hence, the ADOPs of the different 
single-baseline GNSS models can be ranked as

$$
\mathrm{ADOP}^{\mathrm{GFi}} \leq \mathrm{ADOP}^{\mathrm{SR}-\mathrm{LT}} \leq \mathrm{ADOP}^{\mathrm{MR} / \mathrm{SR}-\mathrm{ST}} \leq \mathrm{ADOP}^{\mathrm{GFr}}
$$

For each of the GNSS models considered, the ADOP is given as function of the measurement precision for code and phase, the number of satellites tracked, the number of frequencies used, the number of epochs used and the ionosphere weighting factor. By means of the ionosphere weighting factor, short, medium and long baselines are covered too. Also time correlation, cross correlation and satellite-dependent weighting have been included. Table 8 summarizes the closed-form ADOP expressions derived in this paper. These expressions not only show clearly the relation between different GNSS models, but they also provide a deep understanding and insight into the various factors contributing to ambiguity resolution and their relation. Hence, they can be easily used to study the effect on ambiguity resolution of new or changing measurement scenarios. It should be emphasized that the closed-form expressions presented are not restricted to GPS only; they hold true for any generic multi-frequency GNSS. Hence, they apply to GPS, modernized GPS, Galileo or other future systems of this kind. As a main conclusion, the easy-to-use ADOP expressions allow one to predict the ambiguity success rate for a wide variety of different singlebaseline measurement scenarios.

Open Access This article is distributed under the terms of the Creative Commons Attribution Noncommercial License which permits any noncommercial use, distribution, and reproduction in any medium, provided the original author(s) and source are credited.

\section{References}

Barrena V, Colmenarejo P (2002) Ambiguity dilution of precision for radio frequency based relative navigation determination. In: Proc 5th Int. ESA Conf on Spacecraft Guidance, Navigation and Control Systems. Frascati, October 22-25, pp 603-607

Bock Y, Gourevitch SA, Counselman CC III, King RW, Abbot RI (1986) Interferometric analysis of GPS phase observations. Manus Geod 11:282-288

Chen X, Vollath U, Landau H, Sauer K (2004) Will Galileo/modernized GPS obsolete network RTK? In: Proc ENC-GNSS 2004. Rotterdam, May 16-19

Chmielewsky MA (1981) Elliptically symmetric distributions: a review and bibliography. Int Statist Rev 49:67-74

Euler HJ, Goad CC (1991) On optimal filtering of GPS dual frequency observations without using orbit information. Bull Géod 65:130143

Goad CC, Yang M (1994) On automatic precision airborne GPS positioning. In: Proc KIS94. Banff, August 30-September 2, pp 131-138

Ji S, Chen W, Zhao C, Ding X, Chen Y (2007) Single epoch ambiguity resolution for Galileo with the CAR and LAMBDA methods. GPS Solutions. doi:10.1007/s10291-007-0057-9

Koch K-R (1999) Parameter estimation and hypothesis testing in linear models, 2nd edn. Springer, Heidelberg
Lee HK, Wang J, Rizos C, Barnes J, Tsujii T, Soon BKH (2002) Analysis of pseudolite augmentation for GPS airborne applications. In: Proc ION GPS-2002. Portland, September 24-27, pp 2610-2618

Lee HK, Wang J, Rizos C (2005) An integer ambiguity resolution procedure for GPS/pseudolite/INS integration. J Geod 79:242-255

Liu GC (2001) Ionosphere-weighted Global Positioning System carrier phase ambiguity resolution. MSc. thesis, Department of Geomatics Engineering, University of Calgary

Mathematical Geodesy and Positioning (MGP), Delft University of Technology (2007) http://www.lr.tudelft.nl/mgp

Milbert D (2005) Influence of pseudorange accuracy on phase ambiguity resolution in various GPS modernization scenarios. Navigation 5(1):29-38

Moore M, Rizos C, Wang J, Boyd G, Mathews K, Williams W, Smith R (2003) Issues concerning the implementation of a low cost attitude solution for an unmanned airborne vehicle (UAV) (2003). In: Proc SatNav 2003. Melbourne, July 22-25, CD-ROM

Odijk D (2000) Weighting ionospheric corrections to improve fast GPS positioning over medium distances. In: Proc ION GPS-2000. Salt Lake City, September 19-22, pp 1113-1123

Odijk D (2002) Fast precise GPS positioning in the presence of ionospheric delays. Publications on Geodesy 52, Netherlands Geodetic Commission, $242 \mathrm{p}$

O'Keefe K, Julien O, Cannon ME, Lachapelle G (2005) Availability, accuracy, reliability and carrier-phase ambiguity resolution with Galileo and GPS. Acta Astron 58:422-434

Priestley MB (1981) Spectral analysis and time series. Probability and mathematical statistics, a series of monographs and textbooks, vols. 1 and 2. Academic, London

Rao CR (1973) Linear statistical inference and its applications, 2nd edn. Wiley, New York

Richtert T, El-Sheimy N (2005) Ionospheric modeling: the key to GNSS ambiguity resolution. GPS World. June 2005, pp 35-40

Schaer S (1994) Stochastische Ionosphärenmodellierung beim 'Rapid Static Positioning' mit GPS. Astronomical Institute, University of Berne, Switzerland

Schaffrin B, Bock Y (1988) A unified scheme for processing GPS dualband phase observations. Bull Géod 62:142-160

Scherzinger BM (2000) Precise robust positioning with inertial/GPS RTK. In: Proc ION GPS-2000. Salt Lake City, September 19-22, pp 155-162

Scherzinger BM (2001) Robust inertially-aided RTK position measurement. In: Proc KIS2001. Banff, June 5-8, 8p CD-ROM

Skaloud J (1998) Reducing the GPS ambiguity search space by including inertial data. In: Proc ION GPS-1998. Nashville, September 15-18, pp 2073-2080

Teunissen PJG (1993) Least-squares estimation of the integer GPS ambiguities. Invited lecture, Sect. IV Theory and Methodology, IAG General Meeting, Beijing

Teunissen PJG (1995) The least-squares ambiguity decorrelation adjustment: A method for fast GPS integer ambiguity estimation. J Geod 70:65-82

Teunissen PJG (1997a) A canonical theory for short GPS baselines. Part I: The baseline precision. J Geod 71:320-336

Teunissen PJG (1997b) A canonical theory for short GPS baselines. Part IV: Precision versus reliability. J Geod 71:513-525

Teunissen PJG (1998) The ionosphere-weighted GPS baseline precision in canonical form. J Geod 72:107-117

Teunissen PJG (1999) An optimality property of the integer leastsquares estimator. J Geod 73:587-593

Teunissen PJG, Kleusberg A (eds) (1998) GPS for geodesy, 2nd edn. Springer, Heidelberg

Teunissen PJG, Odijk D (1997) Ambiguity dilution of precision: definition, properties and application. In: Proc of ION GPS-1997. Kansas City, September 16-19, pp 891-899 
Verhagen S (2005) On the reliability of integer ambiguity resolution. Navigation 5(2):99-110

Vollath U, Sauer K, Amarillo F, Pereira J (2003) Three or four carriershow many are enough? In: Proc of ION GPS/GNSS 2003. Portland, September 9-12, pp 1470-1477

Wang J, Lee HK, Lee YJ, Musa T, Rizos C (2004) Online stochastic modelling for network-based GPS real-time kinematic positioning. In: Proceedings of GNSS 2004, The 2004 International Symposium on GNSS/GPS. Sydney, December 6-8
Wu H (2003) On-the-fly ambiguity resolution with inertial aiding. Master's thesis, University of Calgary, Canada, $171 \mathrm{p}$

Wu F, Kubo N, Yasuda A (2004) Performance evaluation of GPS augmentation using quasi-zenith satellite system. IEEE Trans Aeros Electron Systems 40(4):1249-1261

Xu P, Cannon ME, Lachapelle G (1995) Mixed integer programming for the resolution of the GPS carrier phase ambiguities. Paper presented at IUGG95 Assembly. Boulder, July 2-14 